\title{
Mapping extreme-scale alignments of quasar polarization vectors ${ }^{\star}, \star \star$
}

\author{
D. Hutsemékers ${ }^{1, \star \star \star}$, R. Cabanac ${ }^{2}$, H. Lamy ${ }^{3}$, and D. Sluse ${ }^{1}$
}

\author{
${ }^{1}$ Institut d'Astrophysique et de Géophysique, Université de Liège, Allée du 6 Août 17, B5c, 4000 Liège, Belgium \\ e-mail: hutsemekers@astro.ulg.ac.be \\ 2 Canada France Hawaii Telescope, 65-1238 Mamalahoa Highway, Kamuela, Hawaii 96743, USA \\ 3 BIRA-IASB, Avenue Circulaire 3, 1180 Bruxelles, Belgium
}

Received 29 April 2005 / Accepted 27 June 2005

\begin{abstract}
Based on a new sample of 355 quasars with significant optical polarization and using complementary statistical methods, we confirm that quasar polarization vectors are not randomly oriented over the sky with a probability often in excess of $99.9 \%$. The polarization vectors appear coherently oriented or aligned over huge $(\sim 1 \mathrm{Gpc})$ regions of the sky located at both low $(z \sim 0.5)$ and high $(z \sim 1.5)$ redshifts and characterized by different preferred directions of the quasar polarization. In fact, there seems to exist a regular alternance along the line of sight of regions of randomly and aligned polarization vectors with a typical comoving length scale of $1.5 \mathrm{Gpc}$. Furthermore, the mean polarization angle $\bar{\theta}$ appears to rotate with redshift at the rate of $\sim 30^{\circ}$ per Gpc. The symmetry of the the $\bar{\theta}-z$ relation is mirror-like, the mean polarization angle rotating clockwise with increasing redshift in North Galactic hemisphere and counter-clockwise in the South Galactic one. These characteristics make the alignment effect difficult to explain in terms of local mechanisms, namely a contamination by interstellar polarization in our Galaxy. While interpretations like a global rotation of the Universe can potentially explain the effect, the properties we observe qualitatively correspond to the dichroism and birefringence predicted by photon-pseudoscalar oscillation within a magnetic field. Interestingly, the alignment effect seems to be prominent along an axis not far from preferred directions tentatively identified in the Cosmic Microwave Background maps. Although many questions and more particularly the interpretation of the effect remain open, alignments of quasar polarization vectors appear as a promising new way to probe the Universe and its dark components at extremely large scales.
\end{abstract}

Key words. quasars: general - polarization - large-scale structure of Universe - dark matter - cosmology: observations

\section{Introduction}

Considering a sample of 170 optically polarized quasars with accurate linear polarization measurements, Hutsemékers (1998; hereafter Paper I) discovered that quasar polarization vectors are not randomly oriented over the sky as naturally expected. Indeed, in some regions of the three-dimensional Universe (i.e. in regions delimited in right ascension, declination and redshift), the quasar polarization position angles appear concentrated around preferential directions, suggesting the existence of very large-scale coherent orientations - or alignments - of quasar polarization vectors.

Mainly because the polarization vectors of objects located along the same line of sight but at different redshifts are not accordingly aligned, possible instrumental bias and

\footnotetext{
* Based on observations collected at the European Southern Observatory (ESO, La Silla and Paranal).

$\star \star$ Table A.1 is only available in electronic form at the CDS via anonymous ftp to cdsarc.u-strasbg.fr $(130.79 .128 .5)$ or http://cdsweb.u-strasbg.fr/cgi-bin/qcat?]/A+A/441/915 $\star \star \star$ Chercheur qualifié du FNRS (Belgique).
}

contamination by interstellar polarization are unlikely to be responsible for the observed effect. The very large scales at which these coherent orientations are seen suggest the presence of correlations in objects or fields on spatial scales up to $\sim 1 \mathrm{Gpc}$ at redshift $z \simeq 1-2$, possibly unveiling a new effect of cosmological importance. The interpretation of such large-scale correlations is difficult within the concordance cosmological model and might point at missing ingredients. Ongoing theoretical works (e.g. Das et al. 2005) offer interesting avenues indicating that we might detect a property of dark matter or dark energy.

In order to further study the reality of this alignment effect, we have subsequently carried out a very simple test which consisted in obtaining new polarimetric measurements for quasars located in a region of the sky where the range of their polarization position angles was predicted on the basis of the results of Paper I. These measurements, presented in Hutsemékers \& Lamy (2001; hereafter Paper II), independently confirmed the existence of coherent orientations of quasar polarization vectors in the considered region of the sky. Statistical tests applied to the whole sample of 213 objects indicate that the quasar polarization angles are not uniformly distributed with a 
significance level (i.e. the probability that the observed statistic is due to chance) between $10^{-2}$ and $10^{-3}$. These results were confirmed by Jain et al. (2004) using coordinate-invariant statistics.

In order to have an accurate and complete description of the phenomenon, a large number of new polarization measurements is needed. We have then carried out new polarimetric observations which, combined to recent data from the literature, lead to a new sample of 355 polarized quasars with accurate linear polarization measurements. In the following, we present a comprehensive analysis of this new sample. The characteristics of the sample are described in Sect. 2. The results of the statistical analysis are given in Sect. 3 and maps of the strongest alignments are illustrated in Sect. 4. Possible contamination by interstellar polarization in our Galaxy is discussed in details in Sect. 5. The properties of the alignments are investigated in Sect. 6, and possible interpretations in Sect. 7. A preliminary account of this work is reported in Cabanac et al. (2005).

\section{The new sample}

The polarimetric observations were carried out at the European Southern Observatory (Chile) during five runs in the period August 2000 - October 2003, using the $3.6 \mathrm{~m}$ telescope equipped with EFOSC2 in La Silla and the Very Large Telescope equipped with FORS1 in Paranal. A detailed account of the observations, data reduction and final measurements is given in Sluse et al. (2005), including tests for possible biases in the data. In total, 184 new, mostly $V$-band, polarization measurements were obtained for quasars ${ }^{1}$ located at high galactic latitudes $\left(\left|b_{\mathrm{II}}\right| \geq 30^{\circ}\right)$ in both the North Galactic Pole (NGP) and the South Galactic Pole (SGP) regions. The median uncertainty of the polarization degree is $\simeq 0.25 \%$. The targets were mainly selected from the Véron catalogue (Véron-Cetty \& Véron 2001) and from the Sloan Digital Sky Survey Early and First Data Releases (Schneider et al. 2002, 2003; Reichard et al. 2003). Bright objects were preferred, as well as Broad Absorption Line (BAL), radio-loud and red quasars which are usually more polarized (Hutsemékers et al. 1998, Impey \& Tapia 1990, Smith et al. 2002). Special emphasis has been given to the observation of quasars located in the direction of the previously identified regions of polarization vector alignments, i.e. region A1 located in the NGP region and delimited in (B1950) right ascensions and redshift by $11^{\mathrm{h}} 15^{\mathrm{m}} \leq$ $\alpha \leq 14^{\mathrm{h}} 29^{\mathrm{m}}$ and $1.0 \leq z \leq 2.3$, and region A3 located in the SGP region and delimited by $21^{\mathrm{h}} 20^{\mathrm{m}} \leq \alpha \leq 24^{\mathrm{h}} 00^{\mathrm{m}}$ and $0.7 \leq z \leq 1.5$. These limits in right ascension and redshift were fixed visually in Paper I; it must be emphasized that they only roughly delineate the true regions of polarization vector alignments which, ultimately, should be identified more quantitatively.

The observations were performed with multiple goals in mind: (1) to reassess the significance of the alignments seen towards the SGP region A3 as done in Paper II for the NGP region A1; (2) to increase the sampling over the high-redshift

\footnotetext{
${ }^{1}$ In this paper we use indifferently the terms "quasar" or "QSO" for optically or radio selected quasi-stellar objects.
}

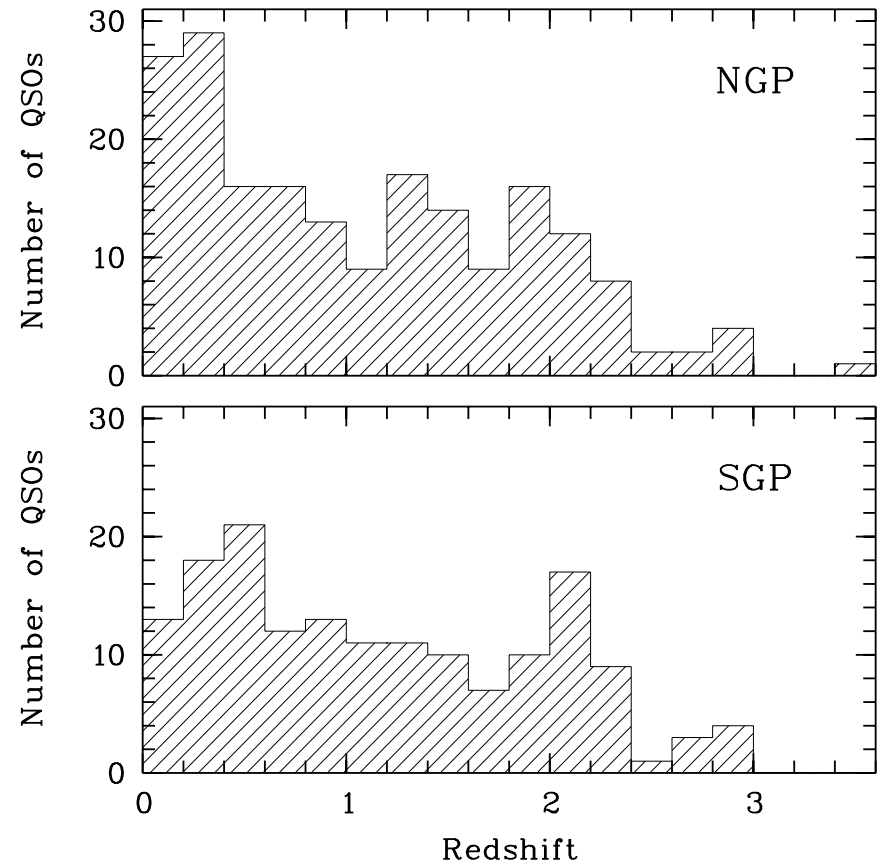

Fig. 1. The redshift distribution of the sample of 355 quasars, illustrated for the NGP and the SGP regions separately.

region A1 where the strongest alignments are measured and to refine its size by investigating the alignment in a sub-region; and (3) to increase the sampling in the foreground regions known to behave differently as shown in Papers I and II.

In the meantime, Smith et al. (2002) have published new, mostly unfiltered, polarization measurements for a sample of $\sim 70$ near-infrared selected QSOs. These objects are added to our sample. Most of them are at redshifts $z \leq 0.5$. We also realized that new redshift measurements were available for a few quasars from the Impey \& Tapia (1990) sample used in Paper I, adding 8 objects to the final sample.

As in Papers I and II, we only consider objects which fulfil the criteria $p \geq 0.6 \%, \sigma_{\theta} \leq 14^{\circ}$, and $\left|b_{\mathrm{II}}\right| \geq 30^{\circ}$, where $p$ is the polarization degree and $\sigma_{\theta}$ the uncertainty of the polarization position angle $\theta$. These constraints ensure that most objects are significantly and intrinsically polarized with little contamination by the Galaxy, and that the polarization position angles are measured with a reasonable accuracy (cf. Paper I, for additional details). If an object has been observed more than once, only the best value is kept i.e. the measurement with the smallest uncertainty $\sigma_{\mathrm{p}}$ on the polarization degree. Objects flagged as contaminated in Sluse et al. (2005) are discarded.

Combining the new data with the sample of 213 objects from Paper II, the final sample of polarized quasars then amounts to 355 objects distributed all over the sky (195 in the NGP region and 160 in the SGP region). The full data set is given in Appendix A. The redshift distribution is illustrated in Fig. 1; it shows a good sampling within the range $0 \leq z \leq 2.4$. The distribution of the polarization degree is illustrated in Fig. 2. 


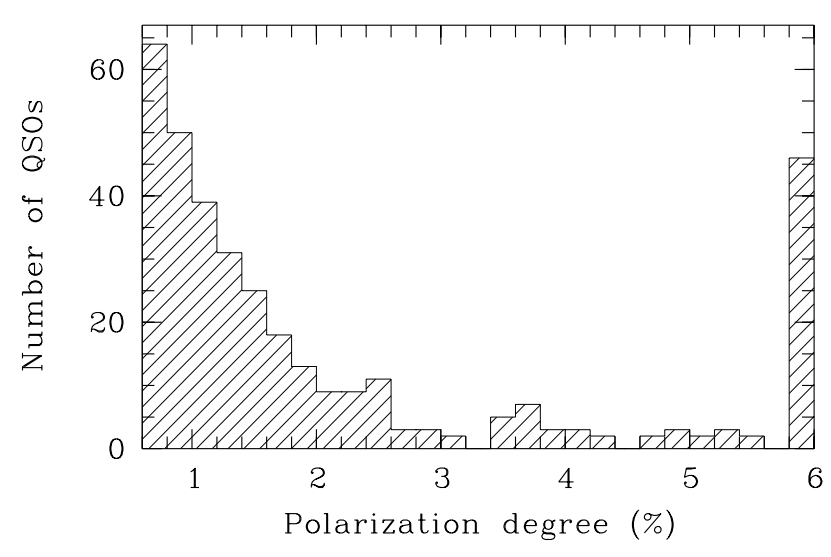

Fig. 2. The distribution of the polarization degree for the sample of 355 quasars. The median is $p \simeq 1.38 \%$. The last bin contains all objects with $p>6 \%$.

\section{Statistical analysis}

\subsection{Local statistics}

In this first series of tests we follow the approach of Paper II, i.e. we test the hypothesis that the polarization position angles ${ }^{2}$ of quasars located in a given region of the sky preferentially lie in the interval $\left[\theta_{1}-\theta_{2}\right]$ instead of being uniformly distributed. This angular sector as well as the region of the sky are selected prior to the new observations, namely on the basis of the results of Paper I. The polarization position angles are measured for a sample of quasars different from that one at the origin of the detection of the effect. To test the null hypothesis of uniform distribution of circular data against the alternative of sectoral preference, we use a simple binomial test (e.g. Lehmacher \& Lienert 1980; Siegel 1956). If $P_{\mathrm{A}}$ is the probability under the null hypothesis that a polarization angle falls in the angular sector $\left[\theta_{1}-\theta_{2}\right]$, then $P_{\mathrm{A}}=\Delta \theta / 180^{\circ}$ where $\Delta \theta=\left(\theta_{2}-\theta_{1}\right)$ mod 180. If $N$ denotes the number of polarization angles falling in $\left[\theta_{1}-\theta_{2}\right]$ out of $N_{0}$ measurements in a given region of the sky, $N$ has a binomial distribution under the null hypothesis and the probability to have $N_{\star}$ or more polarization position angles in $\left[\theta_{1}-\theta_{2}\right]$ is

$P_{\text {bin }}=\sum_{l=N_{\star}}^{N_{0}}\left(\begin{array}{c}N_{0} \\ l\end{array}\right) P_{\mathrm{A}}^{l}\left(1-P_{\mathrm{A}}\right)^{N_{0}-l}$.

The results of the test are given in Table 1. For region A1 we essentially repeat the analysis of Paper II with additional data: out of 40 quasars $^{3}$ in region $\mathrm{A} 1,27$ have their polarization angles in the predicted range $\left[146^{\circ}-46^{\circ}\right]\left(\Delta \theta=80^{\circ}\right.$; this range has been defined in Paper I). The hypothesis of a uniform distribution of the polarization position angles is rejected at the $0.3 \%$ level

\footnotetext{
2 The polarization position angle (or polarization angle) $\theta$ is expressed in degrees from $0^{\circ}$ to $180^{\circ}$ and counted from North to East. Polarization vectors (pseudo-vectors in fact) refer to segments of arbitrary length or normalized on the polarization degree, centered at the object position and with a direction fixed by the polarization angle.

3 Although B1222+228 and B1246-047 have recent, better, measurements reported in Table A.1, these objects were already considered in Paper I and are then discarded from the new sample.
}

Table 1. Binomial statistics.

\begin{tabular}{lccccc}
\hline \hline \multirow{2}{*}{ Region } & & \multicolumn{2}{c}{ New sample } & \multicolumn{2}{c}{ Full sample } \\
& & $N_{\star} / N_{0}$ & $P_{\text {bin }}$ & $N_{\star} / N_{0}$ & $P_{\text {bin }}$ \\
\hline \multirow{2}{*}{ 1 } & $p \geq 0.6 \%$ & $27 / 40$ & $2.8 \times 10^{-3}$ & $42 / 56$ & $3.3 \times 10^{-6}$ \\
& $p \geq 1.0 \%$ & $15 / 22$ & $2.1 \times 10^{-2}$ & $27 / 34$ & $3.4 \times 10^{-5}$ \\
& $p \geq 2.0 \%$ & $5 / 6$ & $6.6 \times 10^{-2}$ & $7 / 8$ & $1.7 \times 10^{-2}$ \\
& $p \geq 3.0 \%$ & $5 / 5$ & $1.7 \times 10^{-2}$ & $6 / 6$ & $7.7 \times 10^{-3}$ \\
A1+ & $p \geq 0.6 \%$ & $13 / 14$ & $2.2 \times 10^{-4}$ & $17 / 18$ & $1.1 \times 10^{-5}$ \\
& $p \geq 1.0 \%$ & $8 / 8$ & $1.5 \times 10^{-3}$ & $12 / 12$ & $5.9 \times 10^{-5}$ \\
A3 & $p \geq 0.6 \%$ & $14 / 18$ & $4.3 \times 10^{-3}$ & $24 / 29$ & $2.6 \times 10^{-5}$ \\
& $p \geq 1.0 \%$ & $9 / 11$ & $1.3 \times 10^{-2}$ & $17 / 20$ & $2.3 \times 10^{-4}$ \\
& $p \geq 2.0 \%$ & $6 / 7$ & $3.3 \times 10^{-2}$ & $12 / 13$ & $4.6 \times 10^{-4}$ \\
& $p \geq 3.0 \%$ & $6 / 7$ & $3.3 \times 10^{-2}$ & $9 / 10$ & $4.1 \times 10^{-3}$ \\
\hline
\end{tabular}

of significance, which is one order of magnitude smaller than in Paper II. Samples with higher cutoff values of the polarization degree $p$ are also considered. They show similar departures to uniformity, indicating that the observed alignments are not only due to the quasars with the smallest polarization levels. For completeness we also provide in Table 1 the numbers for the full sample, i.e. including the data from Paper I. In this case the probability must be seen with caution since the full sample includes objects at the origin of the detection of the effect.

As pointed out in Paper I and II, the polarization vector alignment seems stronger in the inner part of region $\mathrm{A} 1$. We have then defined a smaller region within $\mathrm{A} 1$, denoted $\mathrm{A} 1+$, and delimited a priori by $12^{\mathrm{h}} 00^{\mathrm{m}} \leq \alpha \leq 13^{\mathrm{h}} 20^{\mathrm{m}}$ and $1.3 \leq$ $z \leq 2.0$. Quasars were observed both inside and outside this region. As seen in Table 1, nearly all objects located in region $\mathrm{A} 1+$ have their polarization angles in the range $\left[146^{\circ}-46^{\circ}\right]$. A comparison with the results for the full A1 region indicates that most of the significance is coming from the inner region $\mathrm{A} 1+$. This supports the fact that this alignment occurs within a well defined region of the sky. At the same time this illustrates the difficulty of properly fixing its border.

One of the goals of the new observations was to confirm the polarization vector alignment in region A3 which is roughly opposite to A1 on the sky. In Paper I, we have noted that the polarization angles of the quasars in region $\mathrm{A} 3$ were between $103^{\circ}$ and $144^{\circ}$. If we consider a realistic $\Delta \theta=80^{\circ}$ as for region $\mathrm{A} 1$, we then expect that the polarization angles of quasars located in region A3 will preferentially fall in the angular sector $\left[84^{\circ}-164^{\circ}\right]$. Out of 18 new polarized quasars in this region, 14 are aligned as expected and the hypothesis of an uniform distribution of the polarization position angles may be rejected at the $0.4 \%$ level of significance in favour of coherent orientation. This confirms the existence of large-scale polarization vector alignments also for those quasars located in the SGP region $\mathrm{A} 3$.

\subsection{Global statistics}

Global statistical tests may be applied to the whole sample to detect coherent orientations of polarization vectors in some regions of the sky. Such tests are described in details in Paper I. 


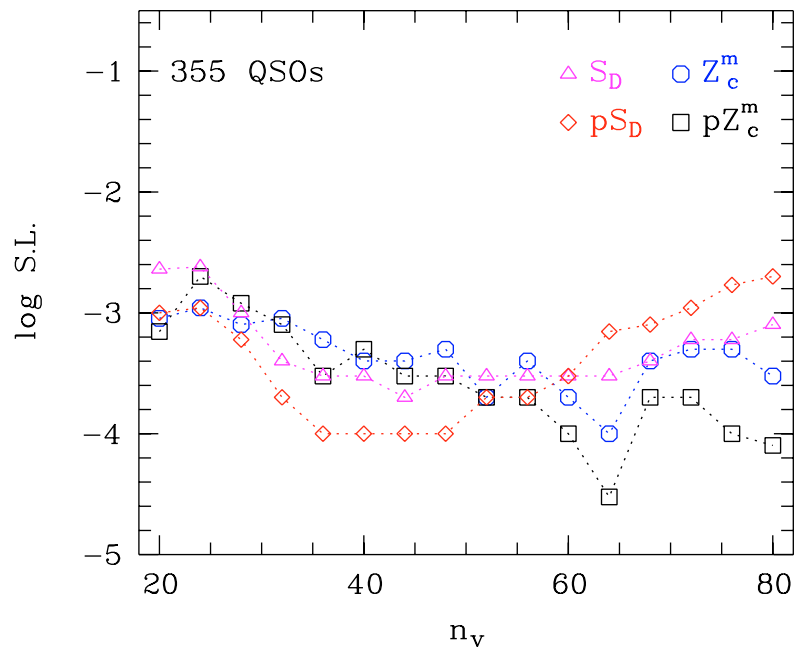

Fig. 3. The logarithmic significance level of the statistical tests applied to the sample of 355 quasars. $n_{\mathrm{v}}$ is the number of nearest neighbours around each quasar and involved in the calculation of the statistics.
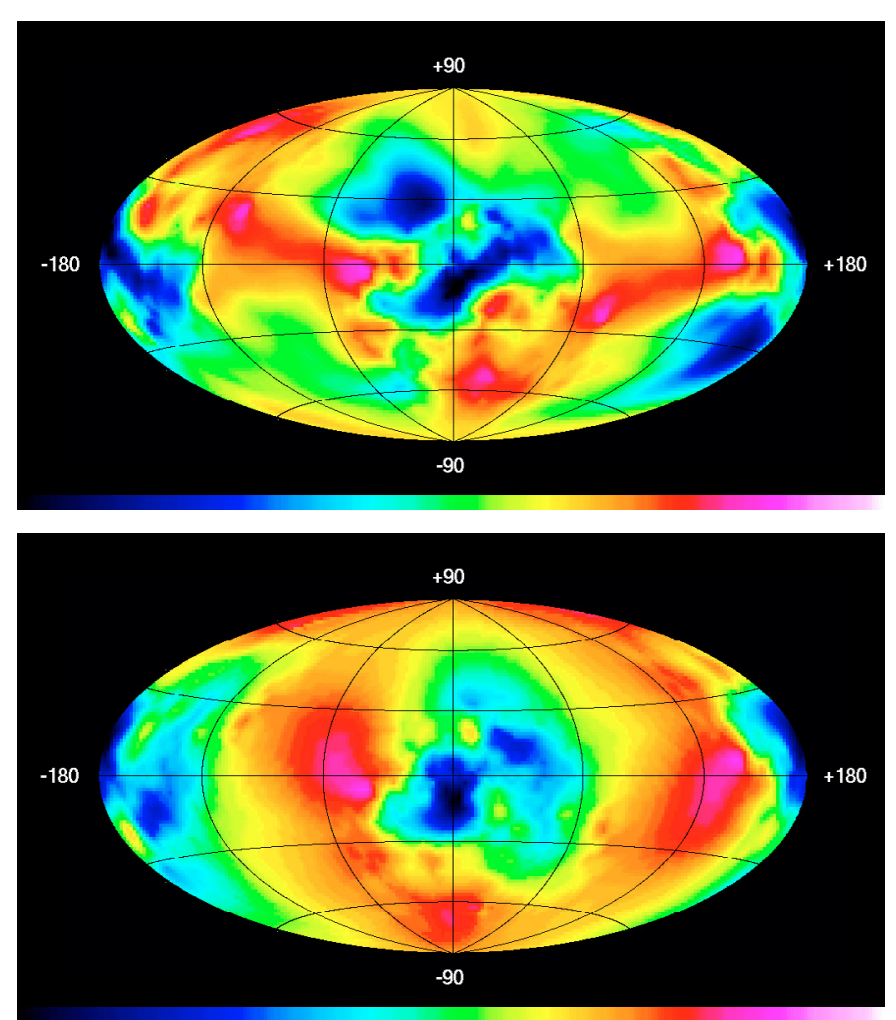

Fig. 4. Hammer-Aitoff projection of the $Z_{c}^{m}$ (top) and $S_{D}$ (bottom) statistics averaged over $n_{\mathrm{V}}=37$ to 43 , as a function of the equatorial coordinates $\alpha_{\mathrm{p}}$ and $\delta_{\mathrm{p}}$ of the northern pole of an arbitrary coordinate system. The less significant the statistics for a given coordinate system or pole position, the darker the corresponding $\left(\alpha_{\mathrm{p}}, \delta_{\mathrm{p}}\right)$ point in the map. Note that $\left(\alpha_{\mathrm{p}}+180^{\circ}, \delta_{\mathrm{p}}\right)$ is equivalent to $\left(\alpha_{\mathrm{p}},-\delta_{\mathrm{p}}\right)$. The full sample of 355 QSOs is used.

Basically, the statistics measure the dispersion of the polarization position angles for groups of $n_{\mathrm{V}}$ nearest neighbours in the 3-dimensional space, summed over all objects in the sample. The significance is evaluated through Monte-Carlo simulations, shuffling angles over positions. A weakness of the tests used in Paper I was their dependency upon the coordinate system. Jain et al. (2004) made them coordinate-invariant by incorporating the parallel transport of polarization vectors.

In the following we consider the $S_{D}$ and the $Z_{c}^{m}$ tests presented in Paper I. Although it is more sensitive, we do not use here the $S$ test because it requires an additional parameter. The tests incorporating parallel transport are denoted $p S_{D}$, and $p Z_{c}^{m}$. The significance levels (SL) of the statistical tests, i.e. the probabilities that the test statistics would have been exceeded by chance only, are computed on the basis of $10^{4}$ permutations. When the significance level is smaller than $10^{-4}$ we used up to $10^{5}$ simulations. Significance levels are given in Fig. 3 for the new sample of 355 quasars against the number of nearest neighbours $n_{\mathrm{v}}$ involved in the calculation of the statistics.

Compared to our previous results (Papers I and II), all the statistical tests indicate a net decrease of the significance level well below $10^{-3}$ - for the new, larger, sample (see also Cabanac et al. 2005 for a comparison). This definitely confirms that quasar polarization vectors are not randomly distributed over the sky but coherently oriented in groups of $\gtrsim 40$ objects, i.e. on Gpc scales at redshift $z \sim 1$. With the increase of the number of objects, we note a shift of the minimum significance level towards higher $n_{\mathrm{v}}$. Tests with and without parallel transport show rather similar results. This is due to the fact that the groups of quasars strongly contributing to the significance are located at low declinations (cf. Sect. 4), i.e. at positions on the celestial sphere where the corrections for parallel transport remain small.

As shown in Paper I, the results of the $S_{D}$ and $Z_{c}^{m}$ tests depend on the adopted coordinate system because the polarization position angles are defined with respect to meridians. When projected onto the equatorial part of the celestial sphere, alignments of polarization vectors are preserved and well detected by the tests. On the contrary, if one chooses a coordinate system with a pole located just in the middle of aligned objects, the polarization angles will range from $0^{\circ}$ to $180^{\circ}$ and no coherent orientation can be detected by the tests. While the parallel transport of polarization vectors solves this problem, it is nevertheless interesting to see for which coordinate systems the significance is extreme. To investigate this, we have computed the statistics for various coordinate systems, each one being characterized by a northern pole of equatorial coordinates $\alpha_{\mathrm{p}}$, $\delta_{\mathrm{p}}$ (see Paper I for details and transformation formulae). The results of these calculations are illustrated in Fig. 4. First, they confirm that the significance is not extreme in the equatorial coordinate system $\left(\delta_{\mathrm{p}}=90^{\circ}\right)$ and that many systems of coordinates do provide more significant statistics, a conclusion already reached in Paper I. Interestingly, the statistics show the lowest significance when using a coordinate system of northern pole $\alpha_{\mathrm{p}} \simeq 0^{\circ}$ and $\delta_{\mathrm{p}} \simeq-10^{\circ}$ (which is equivalent to $\alpha_{\mathrm{p}} \simeq 12^{\mathrm{h}}$, $\delta_{\mathrm{p}} \simeq+10^{\circ}$ ). The location of this pole corresponds to the centers of regions A1 and A3 which are roughly opposite on the $\mathrm{sky}^{4}$. Since putting a polar axis at this location scrambles the

\footnotetext{
${ }^{4}$ When projected onto the sky, the center of region A1 is close to the center of the Local Supercluster (Paper II). The position $\left(\alpha_{p} \simeq 12^{\mathrm{h}}\right.$, $\delta_{\mathrm{p}} \simeq+10^{\circ}$ ) is within a few degrees from the Virgo cluster, known to be at the center of the Local Supercluster (e.g. Vallée 2002).
} 

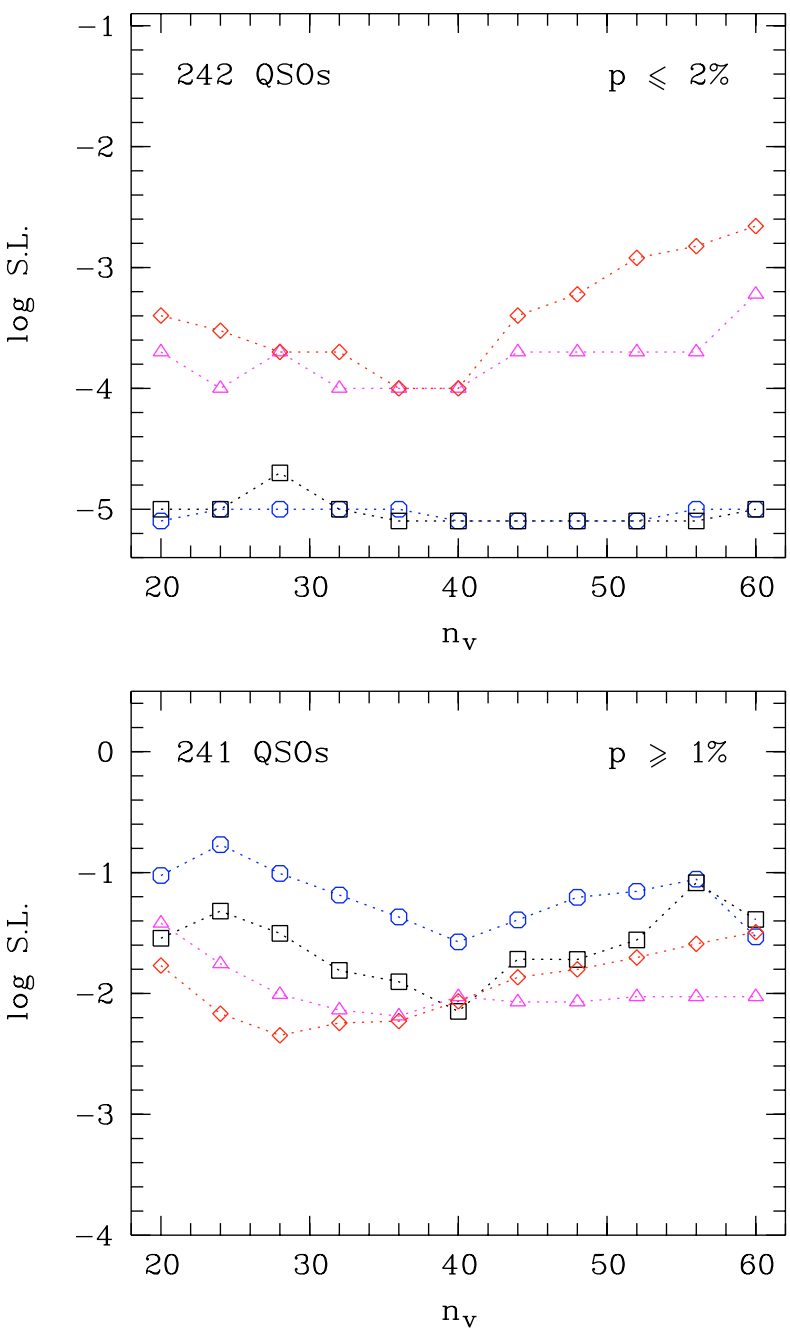

Fig. 5. Same as Fig. 3 but for two sub-samples with different cutoffs of the polarization degree. When SL is smaller than $10^{-5}$ (i.e. unresolved with $10^{5}$ simulations), we arbitrarily fix its value to $\mathrm{SL}=8 \times 10^{-6}$.

most significant alignments, this clearly suggests that regions $\mathrm{A} 1$ and $\mathrm{A} 3$ are major contributors to the global significance. This is independently verified by considering the sample with and without the 183 objects along the "A1-A 3 axis" (as defined in Sect. 4): while a strong departure to uniformity is observed when only those quasars belonging to the $\mathrm{A} 1-\mathrm{A} 3$ region are considered, no significant effect is detected when these objects are removed from the sample.

In Fig. 5, we give the significance levels of the tests considering two sub-samples with cuts on the polarization degree. If only low-polarization $(p \leq 2 \%)$ quasars are considered, the departure to uniformity from the $Z_{c}^{m}$ and $p Z_{c}^{m}$ tests is stronger than in the full sample. For the $p \geq 1 \%$ sub-sample, the departure to uniformity becomes weakly significant. These results are consistent with those obtained by Jain et al. (2004), although the differences we note within our new sample are not as strong as theirs. These differences may indicate that the alignment effect is more efficient for the low polarization quasars than for the high polarization ones. Another reason could be a blurring of the alignments by the high polarization quasars due to the fact that these objects are often variable in both polarization degree and angle (e.g. Impey \& Tapia 1990, a good example being PKS 1216-010 discussed in Sluse et al. 2005). However this behavior seems at odds with the results from local statistics (Table 1) which indicate that high polarization objects are aligned as the low polarization ones, namely in regions $\mathrm{A} 1$ and A3. In fact it is important to realize that cutting at $p \geq 1 \%$ removes proportionally more objects located in the regions of strong alignment $\mathrm{A} 1$ and $\mathrm{A} 3$ than outside these regions, which results in a decrease of the global significance. Inversely, cutting at $p \leq 2 \%$ removes proportionally less objects within these regions, then increasing the global significance. The fact that more low-polarization objects lie in regions A1 and A3 is partly due to the way we have selected the objects. For example, when we got additional data to confirm the alignment in region A1, we preferentially observed BAL QSOs whose polarization levels peak close to $1 \%$ (Hutsemékers \& Lamy 2002; Schmidt \& Hines 1999). Due to such intricate selection effects, the results of the tests applied to sub-samples must be seen with caution.

The same kind of bias occurs when we cut on redshift. Jain et al. (2004) have divided the sample of 213 objects between $z \geq 1$ and $z \leq 1.3$. With $p S_{D}$-type tests, they have noted a stronger alignment effect in the high redshift sub-sample, and no alignment at all in the low redshift one. In fact, when building the sample of 213 objects presented in Paper II, we have mainly added high-redshift objects in region A1, while in the current paper we also add many objects at lower redshifts. As a consequence, when cutting in redshift the new sample of 355 objects, the differences of significance between the low and high redshift sub-samples are not as strong as those reported by Jain et al. (2004). Namely, the tests applied to the new data do not indicate a much higher significance in the high-redshift sample, and a clear signal is seen in the low-redshift one. Cuts on redshift are further discussed in the next section.

\subsection{Semi-global statistics}

Within the sample of 355 quasars, the polarization angles do not appear uniformly distributed, namely when applying a cut on redshift as shown in Fig. 6 (see also Figs. 7 and 8). The isotropy of the histograms are analysed using the HawleyPeebles statistical test which also provides an estimate of the preferred orientations.

The Hawley-Peebles Fourier method (Hawley \& Peebles 1975; see also Paper I) is based on fitting the observed distribution by a model of the form $N\left(\theta_{i}\right)=\bar{N}\left(1+\Delta_{1} \cos 2 \theta_{i}+\right.$ $\left.\Delta_{2} \sin 2 \theta_{i}\right)$ where $\bar{N}$ is the mean number of objects per bin (we adopt 18 bins); $\Delta_{1}$ and $\Delta_{2}$ denote the coefficients of the wave model which describe the degree of deviation from isotropy. The probability that the total amplitude $\Delta=\left(\Delta_{1}^{2}+\right.$ $\left.\Delta_{2}^{2}\right)^{1 / 2}$ exceeds some chosen value is computed to be $P_{\mathrm{HP}}=$ $\exp \left(-0.25 n \Delta^{2}\right)$ where $n$ is the number of objects in the sample. The preferred orientation is calculated from $\bar{\theta}=$ $0.5 \arctan \left(\Delta_{2} / \Delta_{1}\right)$. Results are given in Table 2 for the subsamples illustrated in Fig. 6. We also used the Rayleigh test (e.g. Fisher 1993) which is very similar and gives nearly identical results. 


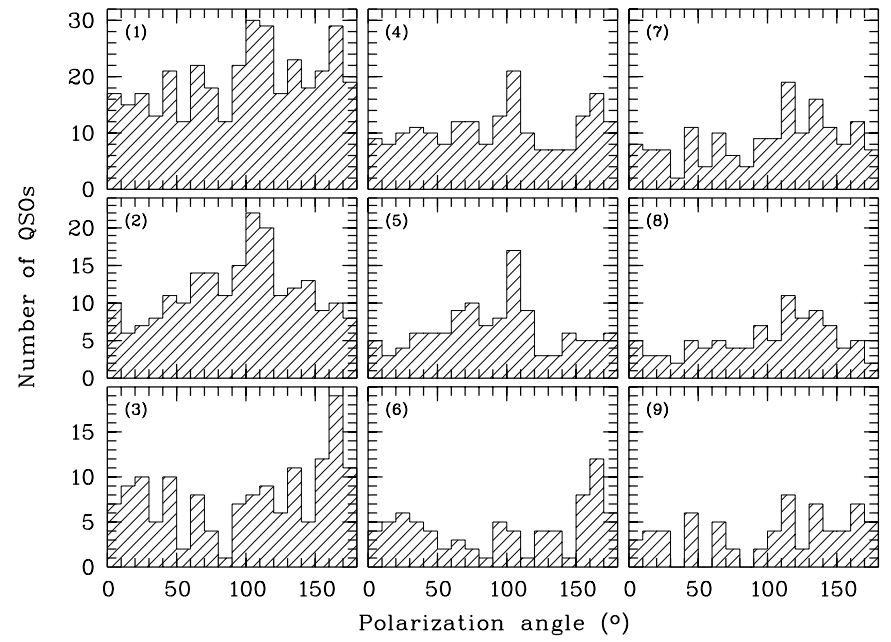

Fig. 6. Distributions of the quasar polarization position angles for different sub-samples. The labels refer to the samples defined in Table 2.

The most remarkable result from Fig. 6 and Table 2 is that both the low and high redshift quasar samples show nonuniform distributions of their polarization angles and different preferred directions. The weak anisotropy in the all- $z$ sample reflects the relative proportion of the various sub-samples. In the NGP region $\left(b_{\mathrm{II}} \geq+30^{\circ}\right)$, the all- $z$ sample is essentially randomly oriented while the low- and high- $z$ samples have very different distributions. The situation is less clear for the SGP region $\left(b_{\mathrm{II}} \leq-30^{\circ}\right)$ : while a definite anisotropy is seen in the low$z$ sample, the evidence for a different preferred direction in the high- $z$ sample is weak, possibly due to the smaller sample. It should be emphasized that this behavior does not mean that all quasars at high or low redshifts have their polarization vectors coherently aligned. Indeed, the observed anisotropy is mainly due to the objects located in the regions of alignment which have been preferentially targetted, as verified by running the test after removing these objects.

\subsection{Statistical tests: summary}

The new sample of 355 quasars has been analysed using various, complementary, statistical methods. All of them concur to indicate that quasars polarization vectors are definitely not randomly oriented but coherently oriented over very large spatial scales. With respect to previous work, the probability that the alignments are due to chance is definitely lower, often smaller than $10^{-3}$.

Towards the NGP, we confirm the high significance of the alignment seen in the high-redshift region A1. A significant alignment - with a different preferred direction - is also detected at lower redshift (see also Fig. 7). In the SGP region, we confirm the alignment previously suspected in region A3. These regions appear as major contributors to the global significance.

Due to its restricted extension in redshift and heterogeneous density, the present sampling does not allow us to study the statistical properties of alignment structures over large volumes. Yet we see clear trends for alignments with different preferred
Table 2. Results of the Hawley-Peebles test.

\begin{tabular}{lrcr}
\hline \hline Sample & $n$ & $P_{\mathrm{HP}}$ & $\bar{\theta}\left({ }^{\circ}\right)$ \\
\hline (1) All & 355 & $2.6 \times 10^{-2}$ & 128 \\
(2) $z \leq 1.3$ & 211 & $2.9 \times 10^{-4}$ & 104 \\
(3) $z \geq 1.3$ & 144 & $8.9 \times 10^{-4}$ & 165 \\
(4) $b_{\mathrm{II}} \geq+30^{\circ}$ & 195 & $8.2 \times 10^{-1}$ & 107 \\
(5) $b_{\mathrm{II}} \geq+30^{\circ}, z \leq 1.3$ & 118 & $4.7 \times 10^{-3}$ & 90 \\
(6) $b_{\mathrm{II}} \geq+30^{\circ}, z \geq 1.3$ & 77 & $4.9 \times 10^{-3}$ & 175 \\
(7) $b_{\mathrm{II}} \leq-30^{\circ}$ & 160 & $1.9 \times 10^{-3}$ & 132 \\
(8) $b_{\mathrm{II}} \leq-30^{\circ}, z \leq 1.3$ & 93 & $4.2 \times 10^{-3}$ & 119 \\
(9) $b_{\mathrm{II}} \leq-30^{\circ}, z \geq 1.3$ & 67 & $4.6 \times 10^{-2}$ & 151 \\
\hline
\end{tabular}

directions to occur in well-defined, although loosely delimited, regions of the sky. Further characterization will require a denser and larger sampling.

\section{Maps of the alignments}

In Figs. 7 and 8, we illustrate the regions where the quasar polarization vector alignments are the most significant. As already discussed, the borders of these regions are not clearcut. This is especially true in the SGP region where several quasars with right ascensions between $0^{\circ}$ and $40^{\circ}$ seem to have their polarization vectors aligned too, at least in some redshift ranges. However, because the spatial sampling is still poor out of the line of sight to regions $\mathrm{A} 1$ and $\mathrm{A} 3$, we choose to essentially stick to the limits adopted in Paper I.

Towards the NGP (Fig. 7), polarization vector alignments are seen for both the low and high redshift samples ${ }^{5}$. The average directions are definitely different: $\bar{\theta} \simeq 79^{\circ}$ at low- $z$ and $\bar{\theta} \simeq 8^{\circ}$ at high- $z$ (with $P_{\mathrm{HP}}=3 \times 10^{-3}$ and $P_{\mathrm{HP}}=2 \times 10^{-3}$, respectively). The alignment in the SGP region A3 $(0.7 \leq z \leq 1.5)$ is also clearly seen, including for the higher polarization objects (Fig. 8). The preferred direction is $\bar{\theta} \simeq 128^{\circ}\left(P_{\mathrm{HP}}=6 \times 10^{-5}\right)$. No significant departure to random orientations is seen in the lower or the higher redshift SGP regions. One might suspect in the high- $z$ region an alignment with a preferred direction different from the mid- $z$ one, but it is not significant. However, if we only consider the 15 high- $z$ objects with $p \geqq 1.2 \%$, we have a weak detection with a preferred direction $\bar{\theta} \sim 15^{\circ}$ $\left(P_{\mathrm{HP}}=3 \times 10^{-2}\right)$. More data are clearly needed towards this region of the SGP.

It is important to emphasize that, in both the NGP and the SGP regions, the polarization degree distributions in the different redshift sub-samples do not significantly differ (as verified with two-sample Kolmogorov-Smirnov tests), and that both the lower and higher polarization quasars follow the same trends.

Finally, since regions A1 and A3 are roughly opposite on the sky, we will refer in the following to the regions defined

\footnotetext{
5 The low redshift region was not analysed in Sect. 3.1 because it was not defined a priori (it overlaps but differs from the region A2 defined in Paper I). It is nevertheless interesting to note that out of 43 polarized quasars in that region, 35 have their polarization angle in the range $\left[30^{\circ}-120^{\circ}\right]$ (cf. Fig. 7). This corresponds to $P_{\text {bin }}=2.1 \times$ $10^{-5}$. With $p \geq 1 \%$ ( $\left.p \geq 2 \%\right), 24$ (13) quasars out of 30 (16) have their polarization angle in that range and $P_{\text {bin }}=7.2 \times 10^{-4}\left(1.1 \times 10^{-2}\right)$.
} 

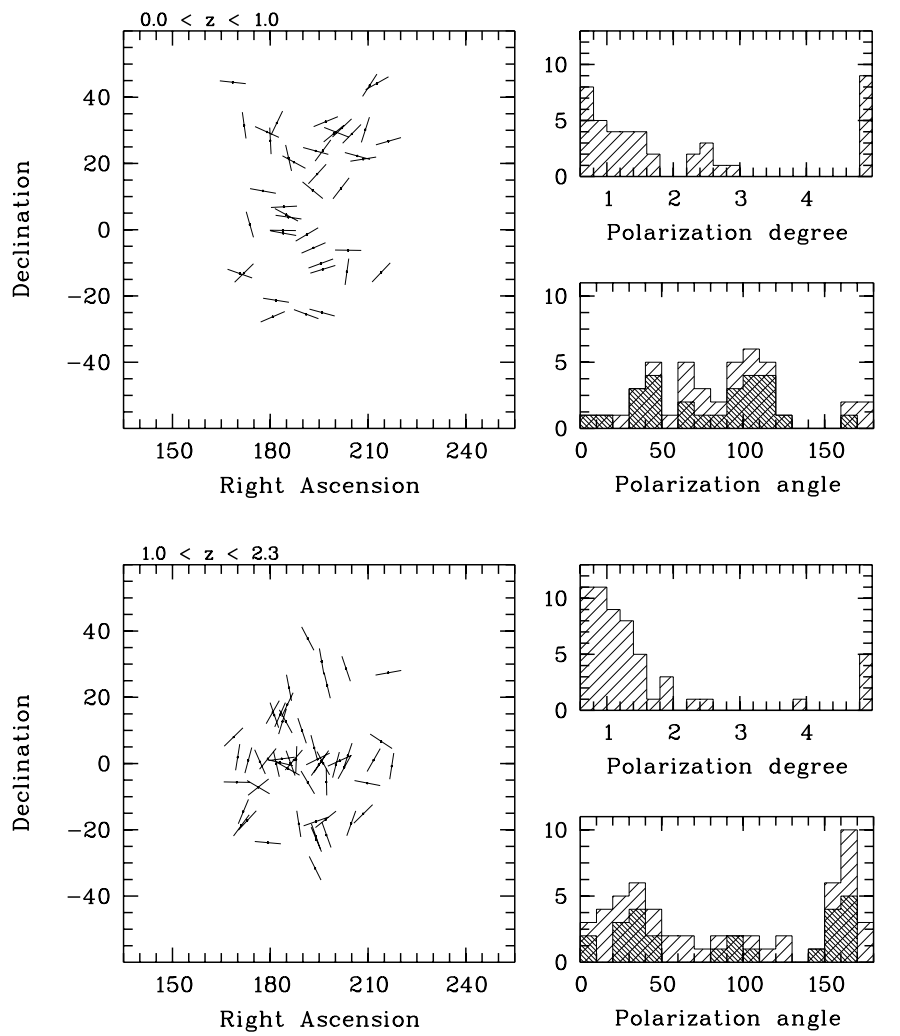

Fig. 7. Maps of quasar polarization vectors in the NGP region, together with the corresponding distributions of polarization degree and angle. The regions illustrated are delimited in right ascension and declination by $168^{\circ} \leq \alpha \leq 218^{\circ}$ and $\delta \leq 50^{\circ}$, and in redshift by $0.0 \leq z<1.0$ (top, 43 objects) and $1.0 \leq z \leq 2.3$ (bottom, 56 objects; region A1). The darker polarization angle histograms refer to quasars with $p \geq 1.2 \%$, that is 2 times higher than the cutoff $p \geq 0.6 \%$ adopted for the full sample.

in right ascension and declination as in Figs. 7 and 8 as to the "A1-A3 axis".

\section{Contamination by interstellar polarization in our Galaxy}

The linear dichroism of aligned interstellar dust grains in our Galaxy produces linear polarization along the line of sight which contaminates to some extent the quasar measurements and may change their polarization angles. Specifically, are the observed alignments due to polarization in our Galaxy? Although this important issue was extensively discussed in Paper I, it is worth to come back on it given our larger sample.

Let us first recall that, whenever possible, we have measured the polarization of field stars located very close to the quasars, on the same CCD frames. If we assume that the field star polarization correctly represents the interstellar polarization affecting more distant objects, then interstellar polarization in our Galaxy was shown to have little effect on the polarization angle distribution of significantly polarized $(p \geq 0.6 \%)$ quasars (Sluse et al. 2005).
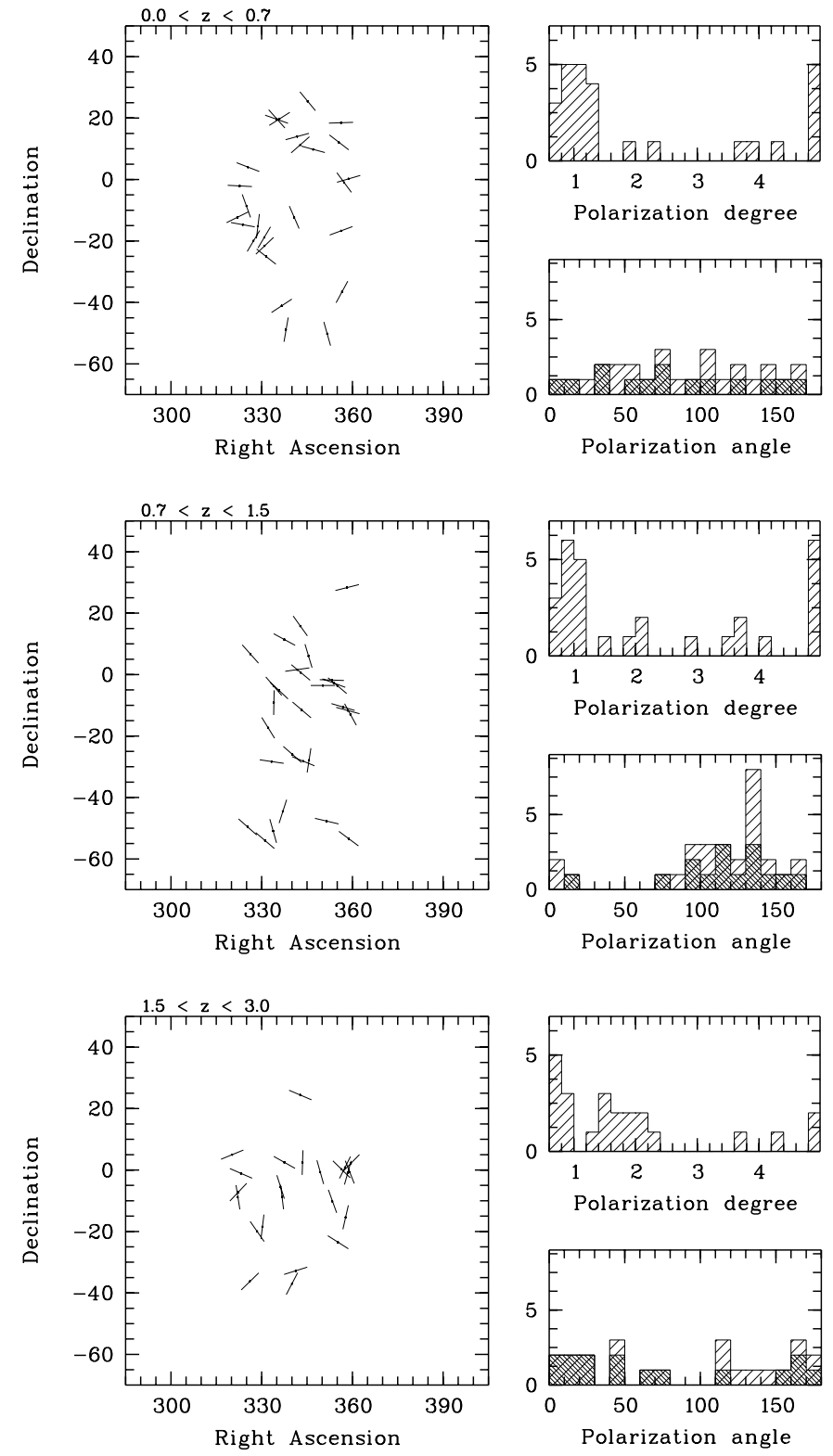

Fig. 8. Maps of quasar polarization vectors in the SGP region, together with the corresponding distributions of polarization degree and angle. The regions illustrated are delimited in right ascension by $320^{\circ} \leq \alpha \leq$ $360^{\circ}$, and in redshift by $0.0 \leq z<0.7$ (top, 27 objects), $0.7 \leq z \leq 1.5$ (middle, 29 objects; region A3) and $1.5<z \leq 3.0$ (bottom, 23 objects). The darker polarization angle histograms refer to quasars with $p \geq$ $1.2 \%$. Right ascensions should be read modulo $360^{\circ}$.

Since accurate field star measurements are not available for every quasar in the sample, we consider in the following the polarization data collected by Heiles (2000) for more than 9000 stars. Our field star polarization measurements are in excellent agreement with these data (Sluse et al. 2005). Figure 9 illustrates polarization maps and distributions for the stars best matching the positions of the quasars represented in Figs. 7 and 8. For each quasar, we plot the angularly closest star on the sky located at a heliocentric distance $d_{\star} \geq 100 \mathrm{pc}$ and with an uncertainty on the polarization angle $\sigma_{\theta_{\star}} \leq 14^{\circ}$; if this star is 

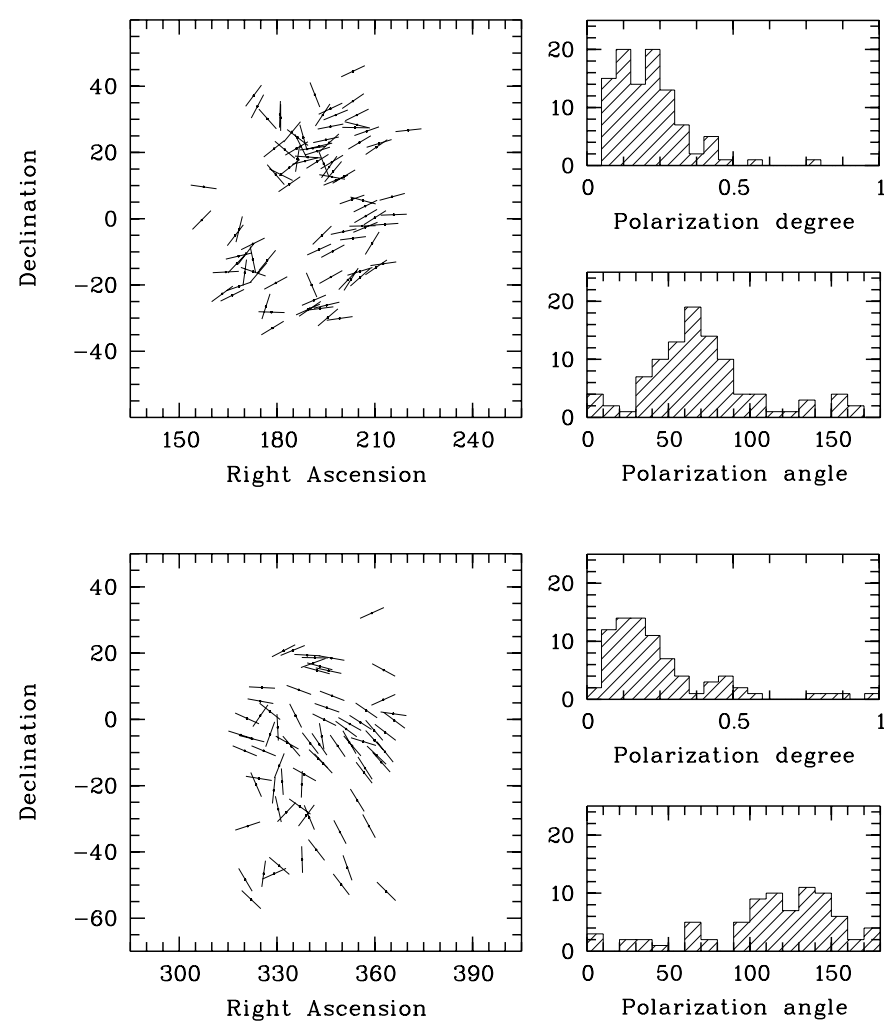

Fig. 9. Maps and corresponding distributions of the interstellar polarization measured from stars matching at best the positions of the quasars illustrated in Figs. 7 and 8 (top: NGP region; bottom: SGP region). Only stars at distances $d_{\star} \geq 100 \mathrm{pc}$ and with a polarization angle uncertainty $\sigma_{\theta_{\star}} \leq 14^{\circ}$ are considered. Since $\sigma_{\theta_{\star}}=28.65 \sigma_{p_{\star}} / p_{\star}$, the latter condition discards stars with $p_{\star} \simeq 0$. The NGP is located at $\alpha=192^{\circ}, \delta=27^{\circ}$ and the SGP at $\alpha=12^{\circ}, \delta=-27^{\circ}$.

already used, we plot the second nearest, etc, making sure that all stars are different. Ideally one should use the most distant stars. However, if we increase the minimum stellar distance, the number density of stars in the catalogue strongly decreases and the mean angular distance to the quasars becomes larger. To keep stars within a few degrees from the quasars, we adopt $d_{\star} \geq 100$ pc as a good compromise. In fact, choosing higher distance cutoffs has little effect on the polarization angle distributions; only the polarization degrees are slightly shifted towards higher values when more distant stars are used. As seen in Fig. 9, the polarization angles are clearly concentrated around two preferred directions: $\bar{\theta}_{\star} \simeq 64^{\circ}$ in the NGP region and $\bar{\theta}_{\star} \simeq 128^{\circ}$ in the SGP region. These mean directions are typical of high galactic latitude regions of the sky (Berdyugin et al. 2004, Sluse et al. 2005) and do not critically depend on the $\alpha, \delta$ bounds.

To compare quasar and stellar polarization angles, we have first computed the difference $\Delta \theta$ between the polarization position angles of a quasar and its nearest star: $\Delta \theta=90^{\circ}-190^{\circ}-$ $\left|\theta-\theta_{\star}\right| \mid$, where $\theta$ refers to the quasar polarization angle and $\theta_{\star}$ to the stellar one. Distributions of $\Delta \theta$ are illustrated in Fig. 10. If quasar polarization vectors are aligned according to interstellar polarization, one may expect a strong clustering at small
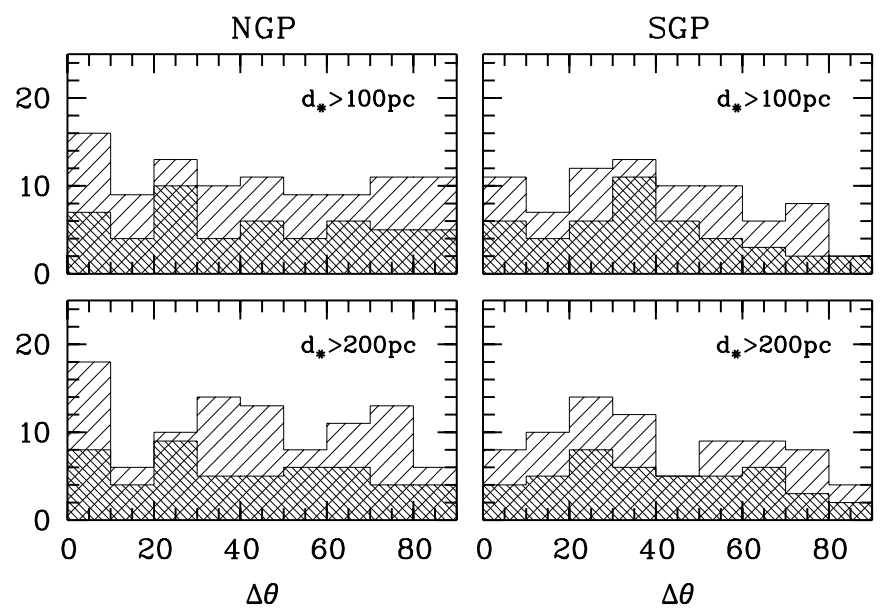

Fig. 10. Distributions of the acute angle $\Delta \theta$ between the polarization position angles of quasars and nearest stars. The objects represented are those considered in Figs. 7-9. The regions of the sky towards the NGP and the SGP are shown separately. Two cutoff in stellar distances are considered: $d_{\star} \geq 100 \mathrm{pc}$ and $d_{\star} \geq 200 \mathrm{pc}$. Darker histograms illustrate distributions of $\Delta \theta$ including only quasars with $p \geq 1.2 \%$.

$\Delta \theta$. Such a clustering is not observed, indicating the absence of significant correlations between quasar and interstellar polarizations (only a weak $2 \sigma$ deviation is seen in the first bin of one of the histograms).

We can also directly compare the trends seen in Fig. 9 to the quasar polarization vector alignments observed in Figs. 7 and 8 . Towards the NGP, the orientation of the alignment in the high-redshift region A1 appears completely different from the direction of the interstellar polarization. But, on the contrary, the mean direction of the lower redshift alignment is rather similar to that one of the interstellar polarization, suggesting that it might be due to polarization by dust grains in our Galaxy, although the distributions somewhat differ and more particularly the peak seen at $\theta \simeq 110^{\circ}$ in the quasar polarization angle distribution. Simple simulations show that, apart from this peak, the clustering in the distribution of low- $z$ quasar polarization angles can be corrected by subtracting a strong (mean $p_{\star} \simeq 0.7 \%$ ) interstellar polarization at $\bar{\theta}_{\star} \simeq 64^{\circ}$. The fact that higher than observed interstellar polarization is needed to randomize the quasar polarization angles is not supported by the observations of distant stars (Berdyugin et al. 2004) nor by the polarization measurements of (a few) field galaxies (Sluse et al. 2005). However, it cannot be rejected since little is known on the interstellar polarization of very distant objects. The fact that interstellar polarization could be at the origin of the low- $z$ alignment is nevertheless difficult to understand since low and high redshift quasars are located on similar lines of sight and then must suffer the same interstellar polarization, at least on average. One might argue that low redshift quasars are systematically less polarized than high redshift ones and then more affected by interstellar polarization. But this interpretation is ruled out by the fact that polarization degrees do not differ in the low 
and high redshift quasar samples ${ }^{6}$ (Fig. 7). Furthermore, highly polarized quasars follow the low- $z$ alignment and low polarization ones follow the high- $z$ alignment. It should be emphasized that very highly polarized quasars do follow the low- $z$ alignment: for example, out of the 7 low- $z$ quasars with $p \geq 7 \%$, 6 have $30^{\circ} \leq \theta \leq 120^{\circ}$. A similar behavior is observed towards the SGP (Fig. 8). The mean orientation of the polarization alignment seen for intermediate redshift quasars coincides with the direction of the interstellar polarization in the SGP, while objects at lower or higher redshifts on the same line of sight show essentially random polarization angle distributions (even weakly oriented at a different $\bar{\theta}$ at high-z). Any correction randomizing the mid- $z$ polarization angle distribution induces a reverse concentration in the distributions of low and high- $z$ quasar polarization angles. And, again, the polarization degrees do not depend on redshift, and the quasars with higher polarization do follow the mid- $z$ alignment (Table 1).

Finally, we have considered pairs of quasars, i.e. quasars at small angular distances from each other, independently of their redshift. If interstellar polarization dominates, both quasars should be similarly affected such that the acute angle $\Delta \theta$ between their polarization angles is expected to cluster at small $\Delta \theta$. Using angular distances less than $1^{\circ}$ and $2^{\circ}$ (smaller distances result in too few quasar pairs), we find no concentration at small $\Delta \theta$, either in the full sample or in the A1-A3 region, or when only considering the low-polarization $(p \leq 2 \%)$ objects more likely to be affected by interstellar polarization (to fix the ideas, with $p \leq 2 \%$, there are 17 quasar pairs with angular separations $\leq 1^{\circ}$ and 61 pairs with angular separations $\leq 2^{\circ}$ among the full sample of 355 quasars).

It is therefore very implausible that interstellar polarization is at the origin of the observed polarization vector alignments. Most probably, there is a small - normal - contamination by interstellar polarization, compatible with field star measurements, and which possibly slightly enhances those intrinsic alignments having a similar orientation. Given that the alignments are characterized by a broad range of polarization angles around a preferred direction, the chance for a coincidence is not small, especially if the mean quasar polarization angle rotates as a function redshift as shown in the next section. The fact that, when cutting at $p \geq 1.2 \%$ in Figs. 7 and 8, quasars with $\theta \simeq 64^{\circ}\left(\theta \simeq 128^{\circ}\right)$ are preferentially removed in the low$z$ NGP region (SGP region) possibly supports this view (cf. also the first bin at $\Delta \theta \leq 10^{\circ}$ in Fig. 10). Spectropolarimetric data are in agreement with this interpretation. Indeed, while a

\footnotetext{
${ }^{6}$ There are several reasons which could have explained such a difference and worth to keep in mind. The first one is that different types of quasars dominate the low and high redshift sub-samples. Indeed, BAL QSOs are rarely detected at $z \leq 1.3$ such that there are proportionally more BAL quasars at high- $z$ than at low- $z$. The reverse is true for radio-loud quasars and the fact that strongly polarized $(p \geq 5 \%)$ quasars are more often found among radio-loud objects is marginally seen in the low- $z$ distribution of the polarization degree (Figs. 7 and 8). Another reason is the fact that, when measuring the polarization either through a given filter or in white light, one samples a bluer region of the quasar rest-frame spectrum for high redshift objects than for low redshift ones. A wavelength dependent quasar polarization would then also appear redshift dependent.
}

significant contamination by interstellar polarization would produce a definite rotation of the polarization angle as a function of wavelength, quasars usually show polarization angles constant (i.e. within a few degrees) with wavelength, including objects located in the regions of alignments (Impey et al. 1995; Ogle et al. 1999; Schmidt \& Smith 2000; Smith et al. 2003; Kishimoto et al. 2004). In the few quasars for which such a rotation is observed, corrections to polarization angles do not exceed $10^{\circ}$ (Kishimoto et al. 2004).

In conclusion, interstellar polarization can definitely not explain the polarization vector alignments seen towards the NGP and more particularly that one observed in the high redshift region A1. Towards the SGP, it is also unlikely that interstellar polarization is at the origin of the observed alignment, but, given the unusual nature of the effect, more data are needed for a definite proof, namely by observing quasars at redshifts $\geq 1.5$ where a different orientation is suspected.

For the sake of completeness, it should be noticed that interstellar dust grains are also linearly birefringent, such that the interstellar medium can be seen as a weak wave-plate (Martin 1974; Lucas 2003). Should quasars be circularly polarized, the interstellar medium may, under some circumstances, align their polarization vectors along a mean direction offsetted with respect to that one of a purely dichroic interstellar medium due to the conversion of circular polarization into linear polarization. While quite appealing, this mechanism cannot explain the quasar polarization vector alignments. Indeed, the retardance is very small, roughly two orders of magnitude smaller than that of a quarter-wave plate (Martin 1972). Also, quasars are not or very weakly circularly polarized (Landstreet \& Angel 1972; Impey et al. 1995), including a few objects belonging to the regions of alignment $\mathrm{A} 1$ and $\mathrm{A} 3$. And, finally, should this effect produce the alignments, it would imply either left-handed or right-handed circular polarization for most quasars in a given region of alignment, i.e. still a high degree of organization on very large spatial scales.

\section{Characterizing the alignment effect}

In this section, we explore some characteristics of the alignment effect with the goal to empirically derive constraints on possible interpretations. We first focus on the redshift dependence of the alignments. Then, we investigate whether quasars with aligned polarization vectors are located along a preferential axis, or not. Finally, we discuss correlations with quasar intrinsic properties.

\subsection{The redshift dependence of the alignment effect}

\subsubsection{Regularly spaced alignments?}

When computing the global statistics in Sect. 3.2, a local statistic $S_{i}$ is defined for each object $i$ and its $n_{\mathrm{v}}$ neighbours. It is evaluated for the original data, $S_{i}^{\star}$, as well as for every simulation. We may then calculate $\left\langle S_{i}\right\rangle$, the average over the whole set of simulations, and $\sigma_{i}$, the corresponding standard deviation, such that the quantity $s_{i} \propto\left|\left\langle S_{i}\right\rangle-S_{i}^{\star}\right| / \sigma_{i}$ provides a measure of the local departure to an uniform distribution of polarization 


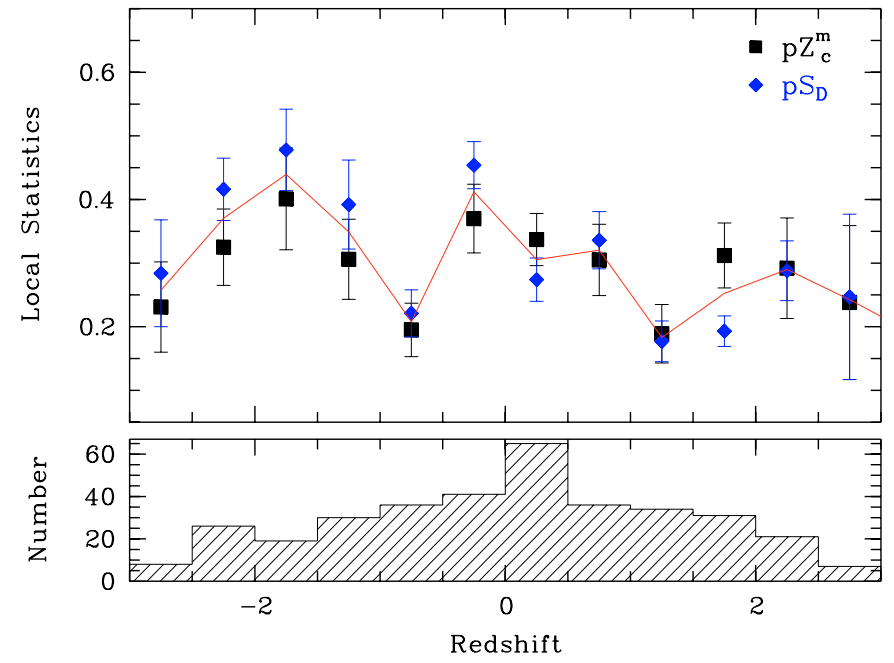

Fig. 11. The local statistics $s_{i}$ of the $p S_{D}$ and $p Z_{c}^{m}$ tests computed with $n_{\mathrm{v}}=40$ are represented here as a function of the redshift. The full sample of 355 quasars is considered. The $s_{i}$, which have been multiplied by a constant factor for convenience, are averaged over redshift bins $\Delta z=0.5$; the error bars represent the uncertainty of the mean. The highest values of $s_{i}$ indicate the strongest departures to uniform distributions of polarization angles. The line joins the mean $s_{i}$ values from the $p S_{D}$ and $p Z_{c}^{m}$ tests. Since the $s_{i}$ are computed for $n_{\mathrm{V}}=40$, the data points are not independent. Redshifts are counted positively for objects located in the North Galactic Cap and negatively for those ones in the South Galactic Cap. The histograms give the number of quasars in each redshift bin.

angles. For the $S$-type tests, only small values of $S_{i}^{\star}$ indicate coherent orientations and $s_{i}$ is set to zero when $S_{i}^{\star}$ is larger than $\left\langle S_{i}\right\rangle$. For the $Z$-type tests, $s_{i}$ is set to zero when $S_{i}^{\star}$ is smaller than $\left\langle S_{i}\right\rangle$ (cf. Paper I for details).

In Fig. 11, we plot the quantity $s_{i}$, averaged over redshift bins, as a function of the redshift. The full sample of 355 quasars is considered. $s_{i}$ is computed from the $p S_{D}$ and $p Z_{c}^{m}$ tests with $n_{\mathrm{v}}=40$. The $S_{D}$ and $Z_{c}^{m}$ tests, not represented here, give similar results. For both statistical tests, the run of $s_{i}$ with redshift shows a cyclic behavior suggesting a regular alternance of regions of aligned and randomly oriented polarization vectors. The minima at $z \simeq 0.7$ towards the SGP and $z \simeq 1.2$ towards the NGP correspond to transition redshifts discussed in previous sections. It must be emphasized that adjacent data points are not independent due to the fact that the statistics $s_{i}$ are evaluated using $n_{\mathrm{v}}=40$ nearest neighbours.

The redshift dependence of the alignment effect is best seen in Fig. 12, when only the $p Z_{c}^{m}$ test and those quasars along the A1-A3 axis (as defined in Sect. 4) are considered. A comoving distance scale is used to emphasize the regular variation of the alignment effect with cosmological distance. This variation appears quasi-periodic, the distance between two extrema being $\sim 1.5 h^{-1}$ Gpc. Such a behavior may clearly constitute an important clue to the interpretation of the alignment effect (Sect. 7). Additional data at high redshift are needed to confirm it. Interestingly enough, a quasi-periodicity in quasar polarization vector alignments, if correctly understood, may potentially constitute a new distance indicator.

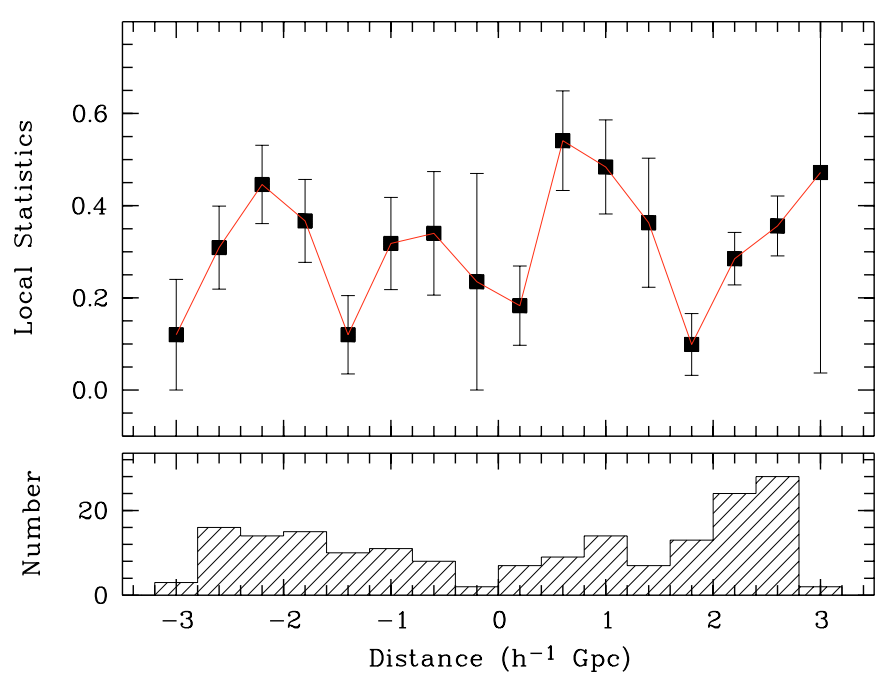

Fig. 12. Same as Fig. 11, except that a comoving distance scale is used and that only those quasars along the A1-A3 axis are considered. Redshifts are transformed into comoving distances using $r=$ $6 \times\left(1-(1+z)^{-1 / 2}\right) h^{-1} \mathrm{Gpc}$, where $h$ is the Hubble constant in units of $100 \mathrm{~km} \mathrm{~s}^{-1} \mathrm{Mpc}^{-1}$. Bin size is $\Delta r=0.4 h^{-1} \mathrm{Gpc}$. Distances are counted positively for objects located in the North Galactic Cap and negatively for those ones in the South Galactic Cap.

\subsubsection{Rotation of the mean direction with redshift?}

Results presented in Sects. 3 and 4 also indicate that the mean polarization angle of quasars changes with redshift. In Fig. 13 we plot the polarization angles of the 355 quasars, slightly averaged over redshift bins, as a function of the redshift. To emphasize possible relationships, each data point is plotted three times in the graph, adding $n \times 180^{\circ}$ to the polarization angles, with $n=0,1,2$. It appears quite clearly that the polarization angles are not randomly distributed over redshifts. Some patterns may be seen as, for example, a continuous decrease of the polarization angle with increasing redshift. The possible relation is more ambiguous around the redshifts $z \simeq 0.7$ towards the SGP and $z \simeq 1.2$ towards the NGP, which correspond to the redshift ranges where no alignment is detected (Fig. 11). Another possible relation could be a decrease of the polarization angle with $z$ in the SGP region $(z<0)$ followed by an increase in the NGP region $(z>0)$.

To investigate more quantitatively possible correlations, we make use of statistical methods which take into account the circular nature of the data; they are described in Fisher (1993). First, we map the redshift onto the circle using $\phi=2 \tan ^{-1} z$, where $z$ is taken to be negative for objects located in the South Galactic Cap and positive for those ones in the North Galactic Cap. As usual, we take into account the axial nature of the polarization angles $\theta$ by multiplying them by a factor 2 . Then we analyse possible correlations between $\phi$ and $\theta$ using the angular-angular correlation coefficients $\widehat{\Pi}_{n}$ and $\widehat{\rho}_{T} . \widehat{\Pi}_{n}$ is a correlation coefficient based on the circular ranks of the $\phi_{i}$ and $\theta_{i}$. It assesses monotone association between $\phi$ and $\theta$. $\widehat{\rho}_{T}$ estimates the linear association between $\phi$ and $\theta$ based on the simple models $\phi=\theta+$ cst or $\phi=-\theta+$ cst. Because it is independent of the scaling of $z$ (including its tranformation into a more physical distance scale), the $\widehat{\Pi}_{n}$ correlation coefficient 


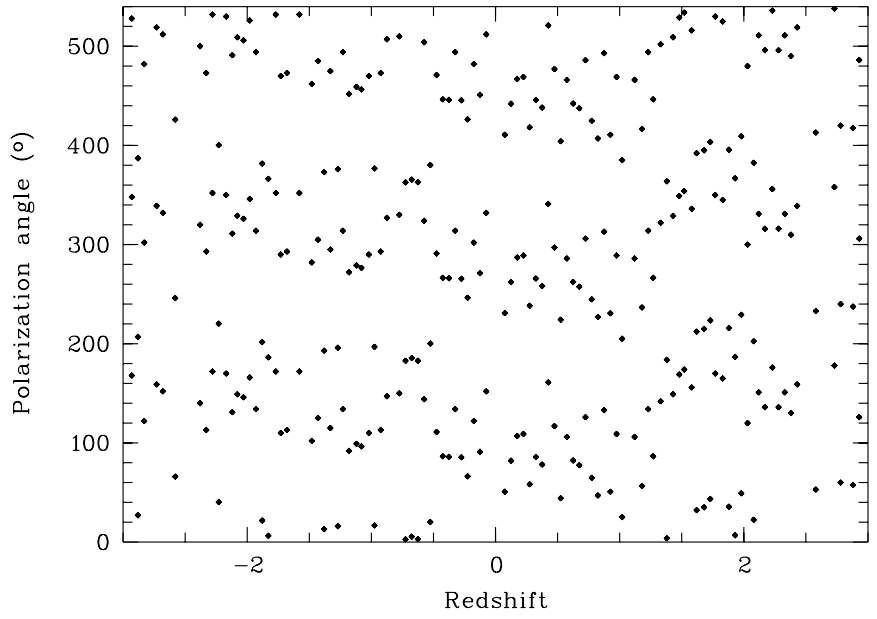

Fig. 13. The quasar polarization angles as a function of the redshift. Redshifts are counted positively for objects located in the North Galactic Cap and negatively for those ones in the South Galactic Cap. Polarization angles are vectorially averaged over redshift bins $\Delta z=0.05$. The full sample of 355 quasars is used. To facilitate detection of patterns, each data point $(z, \theta)$ is replicated at $\left(z, \theta+180^{\circ}\right)$ and $\left(z, \theta+360^{\circ}\right)$.

Table 3. Results of correlation tests.

\begin{tabular}{|c|c|c|c|c|c|}
\hline Sample & & $\widehat{\rho}_{T}$ & $P_{\rho}$ & $\widehat{\Pi}_{n}$ & $P_{\pi}$ \\
\hline $355 \mathrm{~S} 1$ & eq & -0.0254 & $3 \times 10^{-4}$ & -0.0294 & $6 \times 10^{-5}$ \\
\hline $183 \mathrm{~S} 1$ & eq & -0.0702 & $<1 \times 10^{-5}$ & -0.0770 & $<1 \times 10^{-5}$ \\
\hline $129 \mathrm{~S} 1$ & eq & -0.1113 & $<1 \times 10^{-5}$ & -0.1224 & $<1 \times 10^{-5}$ \\
\hline $355 \mathrm{~S} 2$ & eq & -0.0288 & $5 \times 10^{-5}$ & -0.0304 & $3 \times 10^{-5}$ \\
\hline $183 \mathrm{~S} 2$ & eq & -0.0201 & $3 \times 10^{-2}$ & -0.0304 & $4 \times 10^{-3}$ \\
\hline $129 \mathrm{~S} 2$ & eq & -0.0124 & $2 \times 10^{-1}$ & -0.0132 & $2 \times 10^{-1}$ \\
\hline $355 \mathrm{~S} 1$ & $\mathrm{sg}$ & -0.0236 & $3 \times 10^{-4}$ & -0.0188 & $1 \times 10^{-3}$ \\
\hline $183 \mathrm{~S} 1$ & sg & -0.0572 & $2 \times 10^{-5}$ & -0.0488 & $6 \times 10^{-5}$ \\
\hline $129 \mathrm{~S} 1$ & $\mathrm{sg}$ & -0.0782 & $5 \times 10^{-5}$ & -0.0801 & $3 \times 10^{-5}$ \\
\hline $355 \mathrm{~S} 2$ & $\mathrm{sg}$ & -0.0202 & $9 \times 10^{-4}$ & -0.0216 & $5 \times 10^{-4}$ \\
\hline $183 \mathrm{~S} 2$ & sg & -0.0201 & $3 \times 10^{-2}$ & -0.0240 & $1 \times 10^{-2}$ \\
\hline $129 \mathrm{~S} 2$ & sg & -0.0075 & $4 \times 10^{-1}$ & -0.0027 & $7 \times 10^{-1}$ \\
\hline
\end{tabular}

is more general. The hypothesis that $\phi$ and $\theta$ are independent is rejected if $\widehat{\Pi}_{n}$ or $\widehat{\rho}_{T}$ differ too much from zero. The probability that a value more different from zero than the observed values of $\widehat{\Pi}_{n}$ and $\widehat{\rho}_{T}$ would occur by chance among uncorrelated $\phi$ and $\theta$ is evaluated on the basis of $10^{5}$ permutations, shuffling the polarization angles over the redshifts.

The results of the statistical analysis are given in Table 3. The full sample of 355 quasars is considered as well as the sample of 183 objects along the A1-A 3 axis. Out of these 183 quasars, a sub-sample of 129 objects with $p \leq 2 \%$ is also considered. Looking at Fig. 13, we have noticed that polarization angles either continuously decrease with increasing redshift, or decrease in the SGP region $(z<0)$ and increase in the NGP one $(z>0)$. Both possibilities are tested by using the polarization angles "as measured" in both the SGP and NGP regions (case $\mathrm{S} 1$ ), or by taking $180^{\circ}-\theta$ instead of $\theta$ for those objects located in the NGP region (case S2). Since the

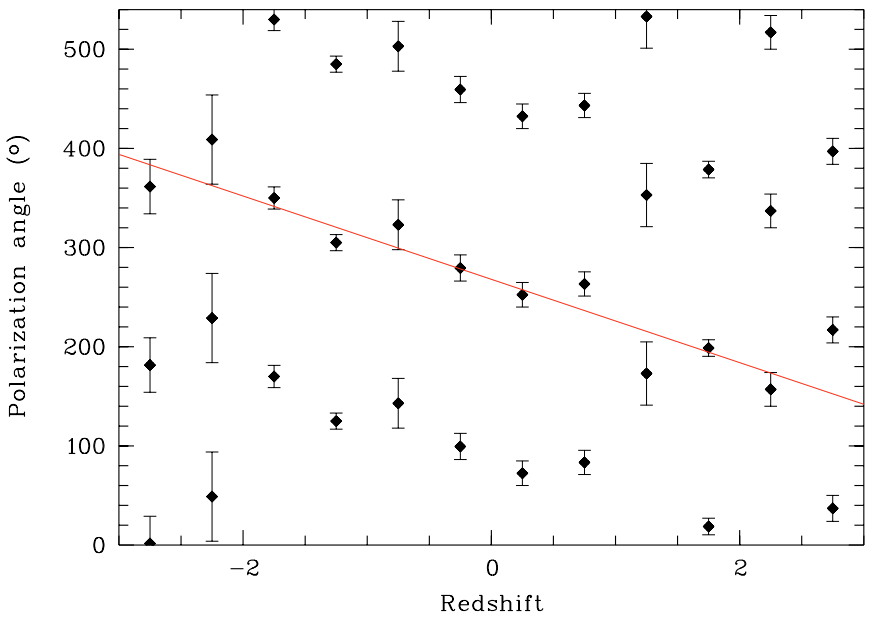

Fig. 14. The quasar polarization angles, vectorially averaged over redshift bins $\Delta z=0.5$, as a function of the redshift. Redshifts are counted positively for objects located in the North Galactic Cap and negatively for those ones in the South Galactic Cap. Only the 183 quasars belonging to the $\mathrm{A} 1-\mathrm{A} 3$ axis are considered here. Error bars represent $68 \%$ angular confidence intervals for the circular mean (Fisher 1993); they must be seen with caution when the number of quasars per redshift bin is small, i.e. at large $z$. Errors are higher at $z \simeq-0.7$ and $z \simeq 1.2$ in agreement with previous results. Data points are replicated at $(z, \theta)$, at $\left(z, \theta+180^{\circ}\right)$ and at $\left(z, \theta+360^{\circ}\right)$ as in Fig. 13. The fitted line is given by $\bar{\theta}=268^{\circ}-42^{\circ} z$ (see text).

mean direction of the alignment in region A1 was found to be roughly parallel to the supergalactic plane (Paper II), the Local Supercluster may constitute a more natural reference frame. We then run the tests with the polarization angles expressed in both the equatorial (eq) and supergalactic ( $\mathrm{sg}$ ) coordinate systems. It essentially appears from Table 3 that the correlation between quasar polarization angles and redshifts is very significant, especially in the case S1 and when only those quasars of the A1-A3 axis are considered. The correlation is significant in both coordinate systems. The tests were also carried out for the sample of 172 quasars obtained when removing the objects which belong to the A1-A3 regions. No correlation was found in that case, again suggesting that the observed effect is mainly due to the objects along the A1-A3 axis.

The $\mathrm{S} 1$ correlation is illustrated in Fig. 14 for the 183 quasars along the A1-A3 axis. It shows a surprisingly clear quasi-linear relation (which is even better defined for the subsample of 129 quasars with $p \leq 2 \%$, in agreement with the results of Table 3$)$. A simple linear regression over the 7 most accurate data points ${ }^{7}$ gives $\bar{\theta}=\left(88^{\circ} \pm 6^{\circ}\right)-\left(42^{\circ} \pm 4^{\circ}\right) z$. This

\footnotetext{
${ }^{7}$ Regression may also be performed using the unbinned data set. In this case the dispersion of the polarization angles is too large and a circular analysis is mandatory. According to Fisher (1993), we may fit the following model to the data: $\bar{\theta}=\bar{\theta}_{0}+\tan ^{-1} \beta z$. The $\theta_{i}$ are assumed to be drawn from a von Mises distribution, with no dependence of the dispersion upon redshift. The maximum likehood estimates of $\beta$ and $\bar{\theta}_{0}$ are $-1.08 \pm 0.27$ and $84^{\circ} \pm 6^{\circ}$, respectively, for the [183-S1eq] sample. This confirms that the correlation between quasar mean polarization angles and redshifts is significant. The analysis with the whole sample of 355 quasars gives similar results.
} 


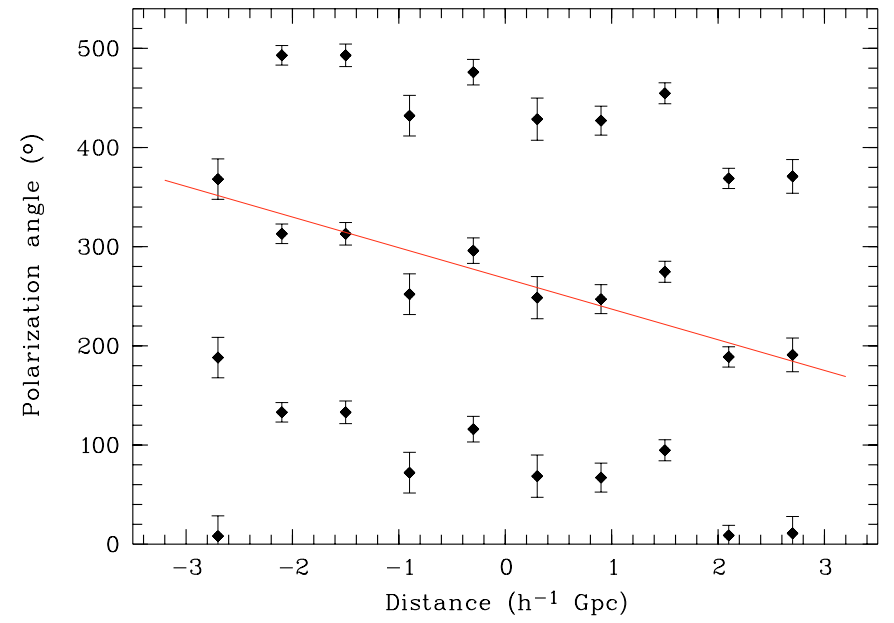

Fig. 15. Same as Fig. 14, except that a comoving distance scale is used (cf. Fig. 12). Bin size is $\Delta r=0.6 h^{-1} \mathrm{Gpc}$. The superimposed line is $\bar{\theta}=268^{\circ}-31^{\circ} r$, where $r$ is the comoving distance in $h^{-1} \mathrm{Gpc}$.

relation reproduces fairly well the preferred directions seen in Figs. 7 and 8. It corresponds to a rotation of roughly $\pm 90^{\circ}$ over the sampled redshift range. It is important to realize that, in general, one may expect a step-like discontinuity at $z=0$, and then need a more complicated fitting of the $\mathrm{S} 1$ correlation. This is due to the way position angles are defined on the celestial sphere. Let us imagine a large-scale structure crossing the observer at $z=0$ and for which we measure a position angle $\theta$ looking towards the NGP. For the same structure, we measure a position angle $-\theta$ looking towards the SGP, which makes a discontinuity in the position angles at $z=0$ (unless $\theta \simeq 0^{\circ}$ or $90^{\circ}$ in the adopted coordinate system). Furthermore, both the slope and the constant of the linear fit depend on the coordinate system. This problem can be partially overcome by parallel transporting the polarization vectors at a given location. More precisely, we may parallel transport the polarization vectors at the position $\left(\alpha_{c}, \delta_{c}\right)$ for those quasars located in the NGP region and $\left(\alpha_{c}+180^{\circ},-\delta_{c}\right)$ for those ones in the SGP region. A reasonable choice is close to the A1-A3 axis, say $\alpha_{c}=180^{\circ}$ and $\delta_{c}=10^{\circ}$. In the S2 case, this makes the $\widehat{\rho}_{T}$ and $\widehat{\Pi}_{n}$ tests fully coordinate invariant, as well as the slope of the linear regression models. In the S1 case, the tests still depend on the coordinates through the discontinuity at $z=0$. With parallel transport, there is a tendency for the S1 correlation to be slightly more significant than in Table 3 , and for the S2 correlation to be slightly less significant. However, we find that the results of the statistical tests and regressions are essentially unchanged, provided that one parallel transports the polarization vectors close to the $\mathrm{A} 1-\mathrm{A} 3$ axis. Results are robust to small changes of $\left(\alpha_{c}, \delta_{c}\right)$. Finally, the rotation of the mean polarization angle is also clearly seen when using comoving distances instead of redshifts (Fig. 15). A linear regression gives $\bar{\theta}=\left(88^{\circ} \pm 5^{\circ}\right)-\left(31^{\circ} \pm 3^{\circ}\right) r$, where $r$ is the comoving distance in $h^{-1} \mathrm{Gpc}$.
The existence of a significant continuous rotation of the mean polarization angle as a function of the redshift ${ }^{8}$ and the symmetry of its dependence are clearly key properties of the alignment effect. While the regions of alignments may look at first glance quite isolated, their properties appear connected on large cosmological distances. The fact that a rotation $\simeq 45^{\circ}$ occurs roughly over the distance between two strong alignments $\left(\sim 1.5 h^{-1} \mathrm{Gpc}\right.$, Fig.12) suggests that both phenomena are probably due to a single mechanism. The simple mirrorlike (S1) symmetry of the $\bar{\theta}-z$ relation is remarkable: rotation is clockwise with increasing redshift in NGP hemisphere and counter-clockwise in the SGP one. This relation is the best defined and the most significant, but a counter-clockwise rotation in both the South and North Galactic Caps (S2 symmetry) cannot be totally excluded, especially if we consider the statistical tests applied to the full sample (Table 3). Also, due to the $180^{\circ}$ uncertainty, several other complicated or asymmetric solutions to the $\bar{\theta}-z$ relation could be imagined. Measurements of quasar polarization angles at redshifts $z \geq 2.5$ are needed to extend and confirm the mirror-like symmetry of the $\bar{\theta}-z$ relation. Moreover, it would allow us to know if the full rotation can exceed $90^{\circ}$ or whether the mean polarization angle oscillates between $0^{\circ}$ and $90^{\circ}$. Finally, it is interesting to note that extrapolating the redshift dependence of the mean polarization angle at $z \simeq 0$ gives $\bar{\theta} \simeq 90^{\circ}$. While this would be an unpleasant coincidence in the equatorial coordinate system, this value corresponds to $\bar{\theta}_{S G} \simeq 0^{\circ}$ in the supergalactic reference frame, which means that the polarization vectors of hypothetical quasars at $z \simeq 0$ should be aligned perpendicular to the supergalactic plane. It is also worth to note that $\bar{\theta}$ at $z=0$ is different from the mean directions of the interstellar polarization (Fig. 9).

\subsection{Is there an alignment axis?}

The fact that the most significant regions of polarization vector alignments are roughly opposite on the sky suggests that they may define an axis in the Universe. The possible coincidence of such an axis with other preferred directions in the sky may provide important clues to the origin of the alignment effect. For example, region A1 is in the direction of Virgo, the center of the Local Supercluster (see also Sect. 3.2), and the A1-A3 axis is not far from the direction of the Cosmic Microwave Background (CMB) dipole $\left(\alpha=168^{\circ}, \delta=-7^{\circ}\right)$. Recent analyses of WMAP data indicate that several largescale anisotropies in the CMB are possibly related to this di-

\footnotetext{
${ }^{8}$ In principle, the redshift dependence of the mean polarization angle may also be a colour effect rather than a distance effect because we are sampling quasar rest-frame spectra at different wavelengths. However spectropolarimetry in the ultraviolet-visible range of (a few) quasars along the A1-A3 axis does not show significant rotation of the continuum polarization angle with wavelength (Impey et al. 1995; Ogle et al. 1999). In general, very few quasars show a rotation of the polarization angle with wavelength. For example, among the 28 polarized quasars studied by Ogle et al. (1999), only 3 display a rotation of the polarization angle, typically $\Delta \theta \sim 20^{\circ}$ over the full ultravioletvisible spectral range.
} 


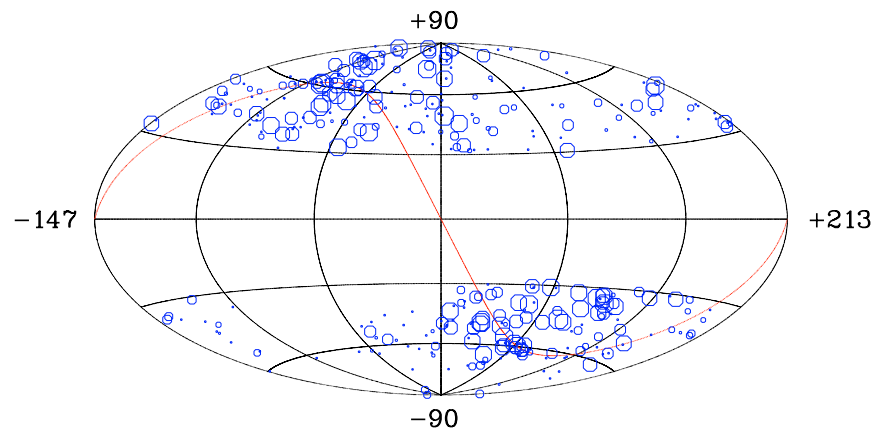

Fig. 16. Hammer-Aitoff projection of the quasar positions on the sky, in Galactic coordinates. The 355 objects are plotted. The radius of the circles is given by $\rho_{i} \propto \exp s_{i}-0.9$, where $s_{i}$ refers the statistic defined in Sect. 6.1 for the $p Z_{c}^{m}$ test and $n_{\mathrm{V}}=40$; the larger the circle the more significant the alignment at that point. The superimposed line gives the location of the celestial equator.
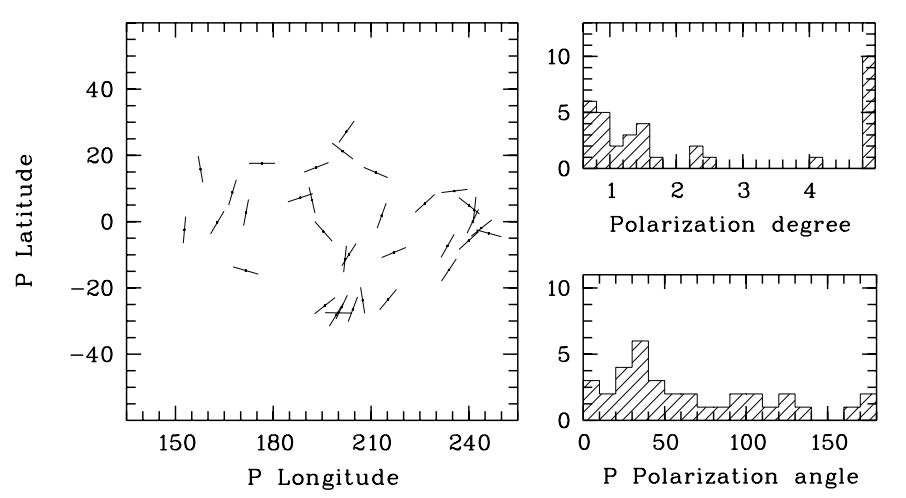

Fig. 17. Maps of quasar polarization vectors and the corresponding distributions of polarization degree and angle for the low-redshift $(z \leq 1)$ objects located in the region of the sky defined in galactic coordinates by $b_{\mathrm{II}} \geq 30^{\circ}$ and by $35^{\circ} \leq l_{\mathrm{II}} \leq 175^{\circ}$ (i.e. in the upper right quadrant of Fig. 16). Positions and polarization angles are projected in a coordinate system of northern pole $\left[\alpha_{p}=0^{\circ}, \delta_{p}=30^{\circ}\right]$ and denoted by "P". The distribution of polarization angles is weakly coherently oriented with $P_{\mathrm{HP}}=2 \times 10^{-2}$ and a preferred direction of $37^{\circ}$ in that coordinate system.

rection (e.g. Tegmark et al. 2003). These possible coincidences are discussed in details by Ralston and Jain (2004).

The evidence for an alignment axis, also suggested from the maps shown in Fig. 4, is best illustrated in Fig. 16 where a dipole-like anisotropy is clearly seen in the distribution of the "most aligned" quasars, as measured from local statistics" ${ }^{9}$. However, this distribution is definitely affected by observational biases. Indeed, in Paper I, we discovered polarization vector alignments for quasars located not far from the celestial equator (the so-called regions A1 and A3), and we subsequently put emphasis on these regions when gathering additional data. Moreover, quasars are often surveyed in

${ }^{9}$ Ralston \& Jain (2004) computed $\left(l_{\mathrm{II}}=266^{\circ}, b_{\mathrm{II}}=61^{\circ}\right)$ for the axis. From Fig. 4 we found $\left(\alpha=180^{\circ}, \delta=10^{\circ}\right)$ which corresponds to $\left(l_{\mathrm{II}}=267^{\circ}, b_{\mathrm{II}}=69^{\circ}\right)$. In Galactic coordinates, the CMB dipole points towards $\left(l_{\mathrm{II}}=264^{\circ}, b_{\mathrm{II}}=48^{\circ}\right)$. equatorial fields which provide the bulk of targets for a southern hemisphere observatory. So, it is not unexpected that the highest quasar densities and the highest significances do appear in these regions. The effect of such intrincate biases on the significance of the axis is difficult to estimate (and is clearly beyond the scope of this paper). However, since preferred axes in the CMB are independently suggested, based on homogeneous data samples and in agreement with the anisotropy seen in Fig. 16, the fact that polarization data are compatible with a possible alignment axis is worth to keep in mind.

A related question is the following: are there really no polarization vector alignments out of the A1-A3 axis? Although measurements are not very numerous, we have tried to identify possible alignments in the upper right quadrant in Fig. 16. Since these objects are close to the celestial north pole, alignments are blurred when polarization angles are measured in equatorial coordinates. We then consider quasar positions and polarization angles in a coordinate system of northern pole $\left[\alpha_{\mathrm{p}}=0^{\circ}, \delta_{\mathrm{p}}=30^{\circ}\right]$. A possible alignment is tentatively identified in Fig. 17; it may constitute a high declination extension to the low-redshift alignment seen in Fig. 7. But, given that very few objects have been measured in these regions (only 46 out of 355 quasars belong to the third of the sky opposite to the regions of highest significance seen in Fig. 16), it is difficult to conclude and the fact that significant polarization vector alignments do or do not exist far from the A1-A3 axis is still to be demonstrated.

\subsection{Relation to quasar intrinsic properties}

Optical polarization is known to be related to other quasar intrinsic properties like spectral type or morphology. In order to understand the alignment effect, it is important to know whether these relations are still valid for those objects in the regions of alignments. A full answer would require a much larger sample and more information on the objects than available in the literature. Some questions have nevertheless been adressed in Papers I and II and are summarized here.

In the high-redshift region of alignment A1, several types of quasars have been observed, namely radio-loud, radio-quiet, and BAL quasars. These distinctions are based on the spectral characteristics of the objects. First, it is important to note that polarization vector alignments are not restricted to one category of objects. In region A1, BAL, non-BAL and radio-loud quasars follow the same alignment, with the same preferred polarization angle. However, possible differences with other types of objects like BL Lac cannot be excluded. Also, it is important to remark that known polarization differences between spectroscopically defined quasar types are not washed out by the alignment effect. For example, the known difference in polarization degree between BAL and non-BAL quasars is still valid in region A1 as demonstrated in Paper II.

Finally, it is interesting to recall that quasar radio polarizations are usually not correlated to optical polarizations, and that radio polarization vectors do not seem to show alignments as the optical polarization vectors do (Paper I, Vallée 2002). 


\section{Possible interpretations}

Possible interpretations of the alignment effect have been discussed in Paper I and II, and more recently by several authors (Jain et al. 2002, 2004; Bezerra et al. 2003; Greyber 2003; Ralston \& Jain 2004). They are further discussed here in the light of the new results.

Since the alignments occur on extremely large scales and appear connected on a sizeable fraction of the known Universe, one must seek for global mechanisms acting at cosmological scales. Possible mechanisms must take into account the fact that the bulk of the measured polarization is intrinsic to the quasars. They may be divided into two broad categories. First, the polarization angles may be closely associated to the morphology of the objects, and the quasar structural axes themselves are aligned on cosmological scales. Alternatively, the polarization angles may be randomly oriented at the source, and modified when the light propagates throughout the Universe. Since both large-scale alignments and regular rotation of the mean orientation must be explained, more than one mechanism may contribute.

If we admit that quasar structural axes are coherently oriented at such large scales, a global rotation of the Universe may be invoked. It would transfer angular momentum to galaxies and quasars during their formation, and, to some extent, correlate their structural axes with the direction of the global rotation (Li 1998). In this case, one would expect the rotation axis to be roughly perpendicular to the A1-A3 direction. While it is not excluded that complex effects like precession could be at the origin of the redshift dependence of the mean orientation, a global rotation would also induce a rotation of the polarization angles as a function of the distance to the source (e.g. Obukhov 2000). The mirror-like symmetry of the $\bar{\theta}-z$ relation illustrated in Figs. 14 and 15 would be accounted for by a rotation axis close to the A1-A3 axis, which is different from the direction needed to produce the alignments. An intermediate position would then be required to explain both effects. From Fig. 15 , we derive a universal angular velocity $\omega_{0} \gtrsim(\pi / 2) H_{0}$ where $H_{0}$ is the Hubble constant, in line with other estimates (Kühne 1997; Obukhov 2000). As a consequence of an inclined axis, alignments should also be observed out of the A1-A3 regions. Furthermore, the rotation of the polarization angle along the line of sight would affect correlations between quasar polarization and structural position angles, at least in some redshift ranges. Interestingly enough, rotating cosmologies have been recently proposed to explain possible anomalies in the CMB (Jaffe et al. 2005). Another possible mechanism for aligning morphological axes could be the effect of magnetic fields coherent over very large scales (Reinhardt 1971; Wasserman 1978; Battaner \& Lesch 2000). Cosmological magnetic fields could make the expansion of the Universe anisotropic (Berera et al. 2004) and then be at the origin of a rotation of the polarization angles (Brans 1975). If polarization vector alignments actually reflect structural alignments, it is nevertheless difficult to explain the alternance of coherently and randomly oriented polarization vectors observed in Fig. 12.

The other possibility is that both the polarization vector alignments and the rotation of the mean polarization angles are due to a mechanism which affects the light on its travel towards the observer. As shown in Paper II, a small amount of polarization added to randomly oriented polarization vectors can be at the origin of coherent orientations of polarization angles ${ }^{10}$ without scrambling too much the relation between polarization and other quasar intrinsic properties. Remarkably, a systematic polarization and a rotation of the polarization angle are predicted by photon-pseudoscalar mixing within a magnetic field, including a quasi-periodic variation of the polarization along the line of sight (e.g. Harari \& Sikivie 1992; Gnedin 1994; Das et al. 2005). Such an oscillation of the polarization added to the quasar intrinsic polarization vectors would appear as a quasiperiodicity in the alignment effect with redshift, in agreement with the results of Sect. 6.1.1. Moreover, an associated rotation of the polarization angles may be expected, as demonstrated by recent simulations (Das et al. 2005). Apparently, photonpseudoscalar mixing has the capability to explain most of the characteristics of the alignment effect, with a coupling constant and a magnetic field strength in agreement with current upper limits. It must be emphasized that this mechanism requires the existence of a - hypothetical - magnetic field organized on cosmological scales. The symmetry of the $\bar{\theta}-z$ relation (Figs. 14 and 15) would then correspond to the symmetry of the magnetic field. Let us finally note that dust grains aligned in a magnetic field can also produce some polarization, but would hardly explain quasi-periodic alignments and a rotation of the mean polarization angle.

Although still hypothetical, photon-pseudoscalar mixing within a magnetic field appears as a promising interpretation, especially because many of the observed characteristics of the alignment effect were predicted, at least qualitatively. Pseudoscalars may be related to dark matter or dark energy, or be ejected by the quasars themselves along with photons (Jain et al. 2002). However, other mechanisms like a global rotation of the Universe cannot be rejected, and should be worked out in more details to see whether or not they can reproduce the observations and constitute viable explanations. In addition to a better spatial sampling namely at higher redshifts, the determination of a possible wavelength dependence of the polarization, the behavior of circular polarization, the relation with quasar morphological axes - especially along the A1-A3 axis - would definitely shed light on the responsible mechanism(s) and more particularly on the photon-pseudoscalar mixing for which rather clear predictions exist (Jain et al. 2002; Das et al. 2005). Observations can also be readily performed

\footnotetext{
10 In fact, if we had in mind to detect the effect of a small systematic polarization, the study of polarization vector alignments is probably one of the most sensitive methods. Indeed, since extragalactic objects are usually intrinsically polarized at various levels (Fig. 2), the addition of a small systematic polarization would be largely undetected, since it only slightly broadens the distribution of the polarization degrees. To some extent, this is also true for low polarization objects (e.g. radio-quiet quasars) because of the errors on the measurements and the subsequent confusion with the polarization degree bias (errors were not taken into account in the simulations of Paper II). On the other hand, a systematic polarization of a few tens of a percent superimposed over randomly oriented polarization vectors do produce a detectable effect in the distribution of the polarization angles.
} 
to demonstrate the existence of a possible preferred alignment axis, and its relation to other tentative anisotropies in the Universe suggested either from the CMB data or from other possible large-scale effects like the - still controversial - Birch effect (Birch 1982; Jain \& Ralston 1999).

\section{Conclusions}

Based on new observations carried out during the period 2000-2003, we have constructed a new sample of quasar polarization measurements in order to further investigate the extreme-scale alignments of quasar polarization vectors discovered in Paper I. The new sample contains 355 polarized quasars, i.e. more than two times the initial sample of 170 objects. Our goal was to firmly reassess the significance of the alignment effect and to empirically derive constraints on possible interpretations.

Using various, complementary, statistical methods, we demonstrate that quasar polarization angles are definitely not randomly oriented over the sky. Polarization vectors appear coherently oriented over very large spatial scales, in regions located at both low and high redshifts and characterized by different preferred directions. These properties make the alignment effect difficult to explain in terms of local mechanisms, like a contamination by interstellar polarization in our Galaxy.

Next, we tried to empirically characterize the effect and more particularly its dependence on redshift. We found a regular alternance of regions of coherently and randomly oriented polarization vectors along the line of sight. We also showed that the mean polarization angle is significantly correlated to redshift, rotating clockwise with increasing redshift in North Galactic hemisphere and counter-clockwise in the South Galactic one. Interestingly enough, the alignment effect seems to be prominent along an axis not far from preferred directions tentatively identified in Cosmic Microwave Background maps.

The fact that polarization vector alignments do occur on extremely large scales and seem connected on a sizeable fraction of the known Universe points towards a global mechanism acting at the scale of the Universe. While several mechanisms like global rotation may, at least partially, explain the alignment effect, we note that the observed behavior remarkly corresponds to the dichroism and birefringence predicted by photon-pseudoscalar oscillation within a magnetic field, suggesting that we might have found a signature of either dark matter or dark energy.

Such interpretations would have profound implications on our understanding of the Universe and then certainly deserve further studies. Fortunately, simple observations, although time consuming, would readily allow us to distinguish between possible interpretations, the alignment effect then providing us with a new tool to probe the Universe and its dark components.

\section{Appendix A: Tables}

In Table A.1 we give the polarization measurements for the full sample of 355 quasars, i.e. B1950 name/coordinates, the redshift $z$, the polarization degree $p$ and its uncertainty $\sigma_{\mathrm{p}}$, the polarization position angle $\theta$ and its uncertainty $\sigma_{\theta}$, and the references to the data. References are coded as follows: (0) Hutsemékers et al. 1998; (1) Berriman et al. 1990; (2) Stockman et al. 1984; (3) Moore \& Stockman 1984; (4) Impey \& Tapia 1990; (5) Impey et al. 1991; (6) Wills et al. 1992; (7) Visvanathan \& Wills 1998; (8) Schmidt \& Hines 1999; (9) Lamy \& Hutsemékers 2000; (10) Smith et al. 2002; (11) Sluse et al. 2005. References [1-6] were considered in Paper I, and [1-9] in Paper II. The eight quasars from Impey \& Tapia (1990) with new redshift measurements and added to the final sample are: B0118-272, B0138-097, B0301-243, B0426-380, $\mathrm{B} 1538+149$, B1606+106, B1749+701, B2206-251. When better polarization measurements are obtained, old values are replaced. Such replacements were indicated in Paper II. In addition, the polarization measurements reported in Sluse et al. (2005) for B1012+008, B1048-090, B1216-010, B1216+069, $\mathrm{B} 1222+228, \mathrm{~B} 1545+210$ and B1617+175 supersede the values used in Papers I and II.

\section{References}

Battaner, E., \& Lesch, H. 2000, Anales de Física, 95, 213

Berdyugin, A., Piirola, V., \& Teerikorpi, P. 2004, A\&A, 424, 873

Berera, A., Buniy, R. V., \& Kephart, T.W. 2004, JCAP, 10, 16

Berriman, G., Schmidt, G. D., West, S. C., \& Stockman, H.S. 1990, ApJS, 74, 869

Bezerra, V. B., Mosquera Cuesta, H.J., \& Ferreira, C.N. 2003, Phys. Rev. D, 67, 084011

Birch, P. 1982, Nature, 298, 451

Brans, C. H. 1975, ApJ, 197, 1

Cabanac, R., Hutsemékers, D., Sluse, D., \& Lamy, H. 2005, ASP Conf. Ser., in press [arXiv: astro-ph/0501043]

Das, S., Jain, P., Ralston, J. P., \& Saha, R. 2005, JCAP, 06, 002

Das, S., Jain, P., Ralston, J. P., \& Saha, R. 2005, [arXiv:hep-ph/0410006]

Fisher, N. I. 1993, Statistical Analysis of Circular Data (Cambridge: Cambridge University Press)

Gnedin, Y. N. 1994, Astron. Astrophys. Transactions, 5, 163

Greyber, H. D. 2003, AIP Conf. Proc., 666, 365

Harari, D., \& Sikivie, P. 1992, Phys. Lett. B, 289, 67

Hawley, D. L., \& Peebles, P. J. E. 1975, AJ, 80, 477

Heiles, C., 2000, AJ, 119, 923

Hutsemékers, D. 1998, A\&A, 332, 410 (Paper I)

Hutsemékers, D., \& Lamy, H. 2001, A\&A, 367, 381 (Paper II)

Hutsemékers, D., \& Lamy, H. 2002, ASP Conf. Ser., 255, 207

Hutsemékers, D., Lamy, H., \& Remy, M. 1998, A\&A, 340, 371

Impey, C. D., \& Tapia, S. 1990, ApJ, 354, 124

Impey, C. D., Lawrence, C. R., \& Tapia, S. 1991, ApJ, 375, 46

Impey, C. D., Malkan, M.A., Webb, W., \& Petry, C. E. 1995, ApJ, 440, 80

Jaffe, T. R., Banday, A. J., Eriksen, H. K., Górski, K. M., \& Hansen, F. K. 2005, ApJ 629, L1

Jain, P., \& Ralston, J. P. 1999, Mod. Phys. Lett., A14, 417

Jain, P., Panda, S., \& Sarala, S. 2002, Phys. Rev. D, 66, 085007

Jain, P., Narain, G., \& Sarala, S. 2004, MNRAS, 347, 394

Kishimoto, M., Antonucci, R., Boisson, C., \& Blaes, O. 2004, MNRAS, 354, 1065

Kühne, R. W. 1997, Mod. Phys. Lett., A12, 2473

Lamy, H., \& Hutsemékers, D. 2000, A\&AS, 142, 451 
Landstreet, J. D., \& Angel, J. R. P. 1972, ApJ, 174, L127

Lehmacher, W., Lienert, G.A. 1980, Biom. J., 22, 249

Li, L.-X. 1998, Gen. Rel. Grav., 30, 497

Lucas, P. W. 2003, JQRST, 79, 921

Martin, P. G. 1972, MNRAS, 159, 179

Martin, P. G. 1974, ApJ, 187, 461

Obukhov, Y. N. 2000, in Cosmic Rotation, ed. Scherfner et al. (Berlin: Wissenschaft und Technik Verlag), 23

Ogle, P. M., Cohen, M. H., Miller, J. S. et al. 1999, ApJS, 125, 1

Moore, R. L., \& Stockman, H.S. 1984, ApJ, 279, 465

Ralston, J. P., \& Jain, P. 2004, Int. J. Mod. Phys., 13, 1857

Reichard, T. A., Richards, G. T., Schneider, D. P., et al. 2003, AJ, 125, 1711

Reinhardt M. 1971, Ap\&SS, 10, 363

Schmidt, G. D., \& Hines, D. C. 1999, ApJ, 512, 125

Schmidt, G. D., \& Smith, P. S. 2000, ApJ, 545, 117

Schneider, D. P., Richards, G. T., Fan, X., et al. 2002, AJ, 123, 567
Schneider, D. P., Fan, X., Hall P. B., et al. 2003, AJ, 126, 2579

Siegel, S. 1956, Nonparametric Statistics (McGraw-Hill)

Sluse, D., Hutsemékers, D., Lamy, H., Cabanac, R., \& Quintana, H. 2005, A\&A, 433, 757

Smith, P. S., Schmidt, G. D., Hines, D. C., Cutri, D. M., \& Nelson, B. O. 2002, ApJ, 569, 23

Smith, P. S., Schmidt, G. D., Hines, D. C., v Foltz, C. B. 2003, ApJ, 593,676

Stockman, H. S., Moore, R.L., \& Angel, J. R. P. 1984, ApJ, 279, 485

Tegmark, M., de Oliveira-Costa, A., Hamilton, A.J. 2003, Phys. Rev. $\mathrm{D}, 68,123523$

Vallée, J. P. 2002, AJ, 1241322

Véron-Cetty, M.-P., \& Véron, P. 2001, A\&A, 374, 92

Visvanathan, N., \& Wills, B. J. 1998, AJ, 116, 2119

Wasserman I. 1978, ApJ, 224, 337

Wills, B. J., Wills, D., Breger, M., Antonucci, R. R. J., \& Barvainis, R. 1992, ApJ, 398, 454 